\title{
STRANGE DUALITY FOR PARABOLIC SYMPLECTIC BUNDLES ON A POINTED PROJECTIVE LINE
}

\author{
TAKESHI ABE
}

ABSTRACT. We prove the strange duality for parabolic symplectic bundles on a pointed projective line.

\section{INTRODUCTION}

Let $C$ be a smooth projective curve over $\mathbb{C}$. Global sections of a line bundle on moduli of vector (or more generally principal $G$-) bundles on $C$ are called generalized theta functions as an analogue to ordinary theta functions on the Jacobian of $C$. In this paper we study the so-called strange duality for symplectic bundles, which is a duality of two vector spaces of generalized theta functions on two different moduli of symplectic vector bundles.

For line bundle $L$ on $C$, a symplectic (resp. orthogonal) bundle with values in $L$ on $C$ is a vector bundle $E$ on $C$ together with a non-degenerate skew-symmetric (resp. symmetric) bilinear form $E \otimes E \rightarrow L$. We denote by $M_{2 r}(C ; L)$ (resp. $\left.N_{2 r}(C ; L)\right)$ the moduli stack of symplectic (resp. orthogonal) bundles with values in $L$ of rank $2 r$ on $C$.

A tensor product of two symplectic bundles is an orthogonal bundle. So we have the tensor-product morphism

$$
\tau: M_{2 r}\left(C ; \mathcal{O}_{C}\right) \times M_{2 s}\left(C ; \omega_{C}\right) \rightarrow N_{4 r s}\left(C ; \omega_{C}\right) .
$$

Let $\mathcal{P}$ be the pfaffian line bundle on $N_{4 r s}\left(C ; \omega_{C}\right)$, that is, the square-root of the determinant line bundle, and let $\Theta$ be the canonical section of $\mathcal{P}$. We have an isomorphism

$$
\tau^{*} \mathcal{P} \simeq \Xi_{M_{2 r}}^{\otimes s} \otimes \Xi_{M_{2 s}}^{\otimes r},
$$

where $\Xi_{M_{2 r}}$ and $\Xi_{M_{2 s}}$ are the determinant line bundles on $M_{2 r}\left(C ; \mathcal{O}_{C}\right)$ and $M_{2 s}\left(C ; \omega_{C}\right)$. The section $\tau^{*} \Theta$ gives rise to the duality map

$$
\mathrm{H}^{0}\left(M_{2 r}\left(C ; \mathcal{O}_{C}\right), \Xi_{M_{2 r}}^{\otimes s}\right)^{\vee} \rightarrow \mathrm{H}^{0}\left(M_{2 s}\left(C ; \omega_{C}\right), \Xi_{M_{2 s}}^{\otimes r}\right) .
$$

We call this map a strange duality map for symplectic bundles. Beauville ([B06]) conjectured that the map (1.2) is an isomorphism. (The strange duality conjecture for ordinary bundles has been proved by Belkale ([Bel08], [Bel07]) and MarianOprea $([\mathrm{M}-\mathrm{O}])$.)

In $[\mathrm{A}]$, the author generalized the strange duality map for symplectic bundles to parabolic symplectic bundles. Let $p^{(1)}, \ldots, p^{(m)}$ be points of $C$. A parabolic symplectic bundle with values in $L$ of rank $2 r$ on the pointed curve $(C ; \vec{p})$ is a symplectic bundle $E$ with values in $L$ of rank $2 r$ on $C$ together with, for each $1 \leq j \leq m$, a flag

$$
\left.E\right|_{p^{(j)}} \supset E_{r}^{(j)} \supset \cdots \supset E_{1}^{(j)} \supset E_{0}^{(j)}=0
$$

by isotropic subspaces $E_{i}^{(j)}$ with $\operatorname{dim} E_{i}^{(j)}=i$. We denote by $M_{2 r}(C, \vec{p} ; L)$ the moduli stack of parabolic symplectic bundles with values in $L$ of rank $2 r$ on $C$.

2000 Mathematics Subject Classification: 14H60, 14D20.

Partially supported by Grant-in-Aid for Young Scientists (B) 17740013. 
When each point $p^{(j)}$ is labeled by an $r$-term non-increasing sequence $\Lambda^{(j)}=(s \geq$ $\lambda_{1}^{(j)} \geq \cdots \geq \lambda_{r}^{(j)} \geq 0$ ) of non-negative integers less than or equal to $s$, we can define a parabolic analogue of the morphism $\tau$ :

$$
\tau_{(C ; \vec{\Lambda})}: M_{2 r}\left(C, \vec{p} ; \mathcal{O}_{C}\right) \times M_{2 s}(C, \vec{p}: \omega(\vec{p})) \rightarrow N_{4 r s}\left(C ; \omega_{C}\right)
$$

We have an isomorphism of line bundles

$$
\tau_{(C ; \vec{\Lambda})}^{*} \mathcal{P} \simeq \Xi_{M_{2 r}}^{(s ; \vec{\Lambda})} \otimes \Xi_{M_{2 s}}^{\left(r ; \vec{\Lambda}^{*}\right)}
$$

where $\Xi_{M_{2 r}}^{(s ; \vec{\Lambda})}$ and $\Xi_{M_{2 s}}^{\left(r ; \vec{\Lambda}^{*}\right)}$ are certain line bundles on the moduli stacks (cf. Definition 2.5). Then as in the non-parabolic case, the section $\tau_{(C ; \vec{\Lambda})}^{*} \Theta$ induces a duality map

$$
\mathrm{H}^{0}\left(M_{2 r}\left(C, \vec{p} ; \mathcal{O}_{C}\right), \Xi_{M_{2 r}}^{(s ; \vec{\Lambda})}\right)^{\vee} \rightarrow \mathrm{H}^{0}\left(M_{2 s}\left(C, \vec{p} ; \omega_{C}(\vec{p})\right), \Xi_{M_{2 s}}^{\left(r ; \vec{\Lambda}^{*}\right)}\right),
$$

which we call the strange duality map for parabolic symplectic bundles. The strange duality conjecture for parabolic symplectic bundles is the following.

Conjecture 1.1. The map (1.3) is an isomorphism.

The main result of $[\mathrm{A}]$ is that if the strange duality conjecture for parabolic symplectic bundles holds for a 3 -pointed $\mathbb{P}^{1}$, then it holds for a generic pointed curve of any genus. In this paper we prove affirmatively the strange duality conjecture for parabolic symplectic bundles for any pointed $\mathbb{P}^{1}$.

1.1. The rank-level duality of conformal blocks. In the theory of conformal blocks, there is a phenomenon called the rank-level duality. Let $\widehat{\mathfrak{g}}$ be an affine Lie algebra, and fix a positive integer $l$, the level. To points $\vec{p}=\left(p^{(1)}, \ldots, p^{(m)}\right)$ of $\mathbb{P}^{1}$ and integrable representations $\vec{\Lambda}=\left(\Lambda^{(1)}, \ldots, \Lambda^{(m)}\right)$ of level $l$ of $\widehat{\mathfrak{g}}$, one can associate a finite dimensional vector space $V_{\mathbb{P 1}}^{\mathfrak{g} \dagger}(\vec{p} ; \vec{\Lambda})$ called the conformal block. The rank-level duality is a duality of certain two conformal blocks. In [NT], Nakanishi and Tsuchiya proved that a certain conformal block of $\widehat{\mathfrak{s l}_{l}}$ of level $r$ is dual to a certain conformal block of $\widehat{\mathfrak{s l}}_{r}$ of level $l$. As mentioned in [NT, §6], one can consider the rank-level duality of conformal blocks of $\widehat{\mathfrak{s p}_{2 r}}$ and $\widehat{\mathfrak{s p}_{2 s}}$. There is a one-to-one correspondence between the set of integrable representations of level $l$ of $\widehat{\mathfrak{s p}_{2 r}}$ and the set of Young diagrams of type $\leq(r, s)$ (cf. §3.3.2). (See $\S 2.1$ for the terminology on Young diagrams.) We identify by this correspondence an integrable representation of level $l$ of $\widehat{\mathfrak{s p}_{2 r}}$ and the corresponding Young diagram of type $\leq(r, s)$. Fix points $\vec{p}=\left(p^{(1)}, \ldots, p^{(m)}\right)$ of $\mathbb{P}^{1}$ and Young diagrams $\vec{\Lambda}=\left(\Lambda^{(1)}, \ldots, \Lambda^{(m)}\right)$ of type $\leq(r, s)$. When both $m$ and $\sum_{j=1}^{m}\left|\Lambda^{(j)}\right|$ are even, we can define the rank-level duality map (cf. $§ 3.4$ )

$$
V_{\mathbb{P}^{1}}^{\mathfrak{s p}_{2 r}^{\dagger}}(\vec{p} ; \vec{\Lambda})_{\text {level }=s}^{\vee} \rightarrow V_{\mathbb{P}^{1}}^{\mathfrak{s p}_{2 s} \dagger}\left(\vec{p} ; \vec{\Lambda}^{*}\right)_{\text {level }=r}
$$

By [L-S], the vector space of global sections of a line bundle on a moduli stack of parabolic $G$-bundle is isomorphic to a conformal block of the affine Lie algebra $\widehat{\mathfrak{g}}$, where $G$ is a simple, simply-connected affine algebraic group and $\mathfrak{g}=\operatorname{Lie}(G)$. For $G=\mathrm{Sp}$, by this isomorphism, the strange duality map for parabolic symplectic bundles is equal to the rank-level duality map of conformal blocks of $\widehat{\mathfrak{s p}}$ (cf. $\S 4$ ).

1.2. Outline of the proof of the main result. The main result of this paper is that the strange duality map for parabolic symplectic bundles on a pointed projective line is an isomorphism. The strange duality and the rank-level duality are equivalent, so we prove the rank-level duality of conformal blocks of $\widehat{\mathfrak{s p}}$. 
We follow closely the line of proof of the rank-level duality of conformal blocks of $\widehat{\mathfrak{s l}}$ in $[\mathrm{NT}]$. We use the degeneration method, and a key fact used in the argument is the compatibility of the factorization and the rank-level duality map. Given a nodal curve $C_{1} \cup C_{2}$ with $C_{1}$ and $C_{2}$ isomorphic to $\mathbb{P}^{1}$ and intersecting only one point $u$. Let $p^{(1)}, \ldots, p^{(a)}$ be points of $C_{1} \backslash\{u\}$, and $q^{(1)}, \ldots, q^{(b)}$ be points of $C_{2} \backslash\{u\}$. The factorization theorem claims that a conformal block on the pointed curve $\left(C_{1} \cup C_{2} ; \vec{p} \cup \vec{q}\right)$ is a direct sum of tensor products of conformal blocks on $\left(C_{1} ; \vec{p} \cup\{u\}\right)$ and $\left.C_{2} ; \vec{q} \cup\{u\}\right)$. By the compatibility of the factorization and the rank-level duality map, we mean that by the factorization the rank-level duality map of conformal blocks of $\widehat{\mathfrak{s p}_{2 r}}$ and $\widehat{\mathfrak{s p}_{2 s}}$ on $\left(C_{1} \cup C_{2} ; \vec{p} \cup \vec{q}\right)$ decomposes as a direct sum of tensor products of rank-level duality maps of conformal blocks of $\widehat{\mathfrak{s p}_{2 r}}$ and $\widehat{\mathfrak{s p}_{2 s}}$ on $\left(C_{1} ; \vec{p} \cup\{u\}\right)$ and $\left.C_{2} ; \vec{q} \cup\{u\}\right)$. This implies that if the rank-level duality maps are isomorphisms on both $\left(C_{1} ; \vec{p} \cup\{u\}\right)$ and $\left(C_{2} ; \vec{q} \cup\{u\}\right)$, then the the rank-level duality map on $\left(C_{1} \cup C_{2} ; \vec{p} \cup \vec{q}\right)$ is an isomorphism.

When $m=4, \Lambda^{(3)}=(1,0, \ldots, 0)$ and $\Lambda^{(4)}=(0, \ldots, 0)$, the conformal blocks appearing in (1.4) have dimension one. So we prove that the rank-level duality map is an isomorphism by showing that it is non-zero (Proposition 6.3). The general case follows from this special case by a degeneration argument similar to that in $[\mathrm{NT}]$.

1.3. This paper is organized as follows.

In Section 2.1, 2.2, 2.3, we prepare the terminology on Young diagrams, flag varieties and Grassmannians. In Section 2.4, 2.5, 2.6, after we define parabolic symplectic bundles and the moduli stack of them and fix notation of line bundles on the moduli stack, we recall the strange duality map for parabolic symplectic bundles formulated in $[\mathrm{A}]$. In Section 3.1 we recall the definition of conformal blocks. In Section 3.2 we recall the factorization theorem of conformal blocks. In Section 3.3 we compute the dimensions of some conformal blocks. In Section 3.4 we formulate the rank-level duality maps of conformal blocks of $\widehat{\mathfrak{s p}_{2 r}}$ and $\widehat{\mathfrak{s p}_{2 s}}$. In Section 3.5 we show the compatibility of the factorization and the rank-level duality map. In Section 4.1 we recall the isomorphism of a space of generalized thetas and a conformal block. In Section 4.2 and 4.3 we show that by the isomorphism the strange duality map is nothing but the rank-level duality map. In Section 5.1 we recall the definition of the KZ connection. In Section 5.2 we show that if the ranklevel duality map is an isomorphism for a pointed projective line, then so is it for all pointed projective lines. In Section 6 we prove that the rank-level duality map is an isomorphism.

\section{Strange DUALITY FOR PARABOLIC SYMPLECTIC BUNDLES}

In this section we recall the formulation of the strange duality for parabolic symplectic bundles.

2.1. Young diagrams. We gather here the terminology on Young diagrams used in this paper.

For positive integers $r$ and $s$, a Young diagram $\Lambda$ is said to be of type $\leq(r, s)$ if the number of rows of $\Lambda$ is less than or equal to $r$ and that of columns of $\Lambda$ is less than or equal to $s$.

By associating to a non-increasing sequence $s \geq \lambda_{1} \geq \cdots \geq \lambda_{r} \geq 0$ of nonnegative integers the Young diagram whose $i$-th row has $\lambda_{i}$ boxes, we obtain a oneto-one correspondence between the set of all $r$-term non-increasing sequences $\lambda_{1} \geq$ $\cdots \geq \lambda_{r} \geq 0$ of non-negative integers with $\lambda_{1} \leq s$ and the set of all Young diagrams of type $\leq(r, s)$. By this correspondence, we use the terms "Young diagram" and "non-increasing sequence of integers" interchangeably. 
For a Young diagram $\Lambda=\left(\lambda_{1} \geq \cdots \geq \lambda_{r}\right)$ of type $\leq(r, s)$, we denote by $\widetilde{\Lambda}$ the Young diagram of type $\leq(s, r)$ that is obtained from $\Lambda$ by interchanging rows and columns. For example, if $\Lambda$ is the Young diagram $(4,2,1)$ of type $\leq(3,4)$, then $\widetilde{\Lambda}$ is the Young diagram $(3,2,1,1)$ of type $\leq(4,3)$.

For a Young diagram $\Lambda=\left(\lambda_{1} \geq \cdots \geq \lambda_{r}\right)$ of type $\leq(r, s)$, we denote by ${ }^{c} \Lambda$ the Young diagram $\left(s-\lambda_{r} \geq s-\lambda_{r-1} \geq \cdots \geq s-\lambda_{1}\right)$ of type $\leq(r, s)$. The Young diagram $\Lambda^{*}$ of type $\leq(s, r)$ is defined to be $\widetilde{\Lambda}$. It is easy to see that if $\Lambda=\left(\lambda_{1} \geq \cdots \geq \lambda_{r}\right)$ and $\Lambda^{*}=\left(\mu_{1} \geq \cdots \geq \mu_{s}\right)$, then

$\left\{\lambda_{1}+r, \lambda_{2}+r-1, \ldots \lambda_{r}+1\right\} \cup\left\{\mu_{1}+s, \mu_{2}+s-1, \ldots, \mu_{s}+1\right\}=\{1,2, \ldots, r+s\}$.

For a Young diagram $\Lambda$, we denote by $|\Lambda|$ the number of boxes in $\Lambda$.

2.2. Symplectic flag varieties. Let $S$ be a scheme, $\mathcal{P}$ a line bundle on $S, \mathcal{E}$ a vector bundle of rank $2 r$ on $S$, and $\pi: \mathcal{E} \otimes \mathcal{E} \rightarrow \mathcal{P}$ a non-degenerate alternate bilinear form. A full flag of $\mathcal{E}$ by isotropic subbundles means a filtration by isotropic subbundles $\mathcal{E} \supset \mathcal{E}_{r} \supset \cdots \supset \mathcal{E}_{1} \supset \mathcal{E}_{0}=0$ with $\operatorname{rank} \mathcal{E}_{i}=i$. (Here by "isotropic" we mean that the restriction of $\pi$ to $\mathcal{E}_{i} \otimes \mathcal{E}_{i}$ is zero.)

Let $\mathbf{F l}(\mathcal{E}) \rightarrow S$ be the flag variety parameterizing full flags of $\mathcal{E}$ by isotropic subbundles. Let

$$
(\mathcal{E})_{\mathbf{F l}(\mathcal{E})} \supset \mathcal{E}_{r} \supset \mathcal{E}_{r-1} \supset \cdots \supset \mathcal{E}_{1} \supset \mathcal{E}_{0}=0
$$

be the universal full flag by isotropic bundles. Given a tuple of integers $\vec{q}=$ $\left(q_{1}, \ldots, q_{r}\right)$, we denote by $\mathcal{O}_{\mathbf{F l}(\mathcal{E})}(\vec{q})($ or simply $\mathcal{O}(\vec{q}))$ the line bundle $\bigotimes_{i=1}^{r}\left(\mathcal{E}_{i-1}^{\perp} / \mathcal{E}_{i}^{\perp}\right)^{\otimes q_{i}}$ on $\operatorname{Fl}(\mathcal{E})$.

Let $\operatorname{Sp}(\mathcal{E})$ be the group scheme over $S$, which parameterizes symplectic automorphisms of $\mathcal{E}$. If $\mathcal{E} \supset \mathcal{E}_{r} \supset \ldots \mathcal{E}_{1} \supset \mathcal{E}_{0}=0$ is a full flag by isotropic subbundles and $\alpha: \mathcal{E} \rightarrow \mathcal{E}$ is a symplectic automorphism, then $\mathcal{E} \supset \alpha\left(\mathcal{E}_{r}\right) \supset \cdots \supset \alpha\left(\mathcal{E}_{1}\right) \supset$ $\alpha\left(\mathcal{E}_{0}\right)=0$ is again a full flag by isotropic subbundles. This gives rises to a left action of $\operatorname{Sp}(\mathcal{E})$ on $\operatorname{Fl}(\mathcal{E})$. The action lifts to the action of each filter $\mathcal{E}_{i}$ of the universal full flag by isotropic subbundles. Hence the vector bundle $\operatorname{pr}_{*} \mathcal{O}_{\mathbf{F l}(\mathcal{E})}(\vec{q})$ on $S$ becomes a (left) $\operatorname{Sp}(\mathcal{E})$-module, where $p r: \operatorname{Fl}(\mathcal{E}) \rightarrow S$ is the projection. The following proposition is well-known.

Proposition 2.1. Assume that $S=$ Spec $k$ with $k$ an algebraically closed field of characteristic zero. The $k$-vector space $\mathrm{H}^{0}(\mathbf{F l}(\mathcal{E}), \mathcal{O}(\vec{q}))$ is non-zero if and only if $q_{1} \geq \cdots \geq q_{r} \geq 0$. By the correspondence

$$
\left(q_{1}, \ldots, q_{r}\right) \leftrightarrow \mathrm{H}^{0}(\mathbf{F l}(\mathcal{E}), \mathcal{O}(\vec{q}))
$$

there is a one-to-one correspondence between the set of all finite dimensional irreducible representations of the symplectic group $\operatorname{Sp}(\mathcal{E})$ and the set of all $\vec{q}=$ $\left(q_{1}, \ldots, q_{r}\right)$ with $q_{1} \geq \cdots \geq q_{r} \geq 0$.

For later use, it would be convenient to prepare here numbering of the filters of a full flag by isotropic subbundles with respect to a Young diagram.

Notation 2.2. Let $\Lambda=\left(s \geq \lambda_{1} \geq \cdots \geq \lambda_{r} \geq 0\right)$ be a Young diagram of type $\leq(r, s)$. Given a full flag of $\mathcal{E}$ by isotropic subbundles

$$
\mathcal{E}_{\bullet}: \mathcal{E} \supset \mathcal{E}_{r} \supset \cdots \supset \mathcal{E}_{1} \supset \mathcal{E}_{0}=0
$$

we put $\mathbb{F}_{i}^{\Lambda}\left(\mathcal{E}_{\bullet}\right):=\mathcal{E}_{l}$ for $s+l-\lambda_{l} \leq i<s+l+1-\lambda_{l+1}$ for $0 \leq i \leq r+s$. 
2.3. Orthogonal Grassmannians. Let $\left(V,(-,-)_{V}\right)$ be a $2 n$-dimensional $k$-vector space with a non-degenerate symmetric bilinear form. We assume that $n$ is even. Let $\mathbf{O G r}_{n}(V)$ be the orthogonal Grassmannian parameterizing isotropic subspaces of $V$ of dimension $n$. Then $\mathbf{O G r}_{n}(V)$ has two connected components $\mathbf{O G r}_{n}^{+}(V)$ and $\mathbf{O} \mathbf{G r}_{n}^{-}(V) ; U$ and $U^{\prime} \in \mathbf{O} \mathbf{G} \mathbf{r}_{n}(V)$ lie in the same connected component if and only if $\operatorname{dim} U \cap U^{\prime}$ is even.

On $\mathbf{O G r}_{n}(V)$, there is a short exact sequence

$$
0 \rightarrow \mathcal{U} \rightarrow V \otimes \mathcal{O}_{\mathbf{O G r}_{n}(V)} \rightarrow \mathcal{Q} \rightarrow 0
$$

given by the universal subbundle $\mathcal{U}$ and the universal quotient bundle $\mathcal{Q}$. There is a unique square root of the line bundle $\operatorname{det} \mathcal{Q}$, which we $\operatorname{denote}$ by $(\operatorname{det} \mathcal{Q})^{\otimes \frac{1}{2}}$.

2.4. The morphism $\mu_{\Lambda}$. Let $\left(E,(-,-)_{E}\right)$ and $\left(G,(-,-)_{G}\right)$ be $k$-vector spaces with a non-degenerate alternate bilinear form of dimension $2 r$ and $2 s$ respectively. We endow the tensor product $E \otimes G$ with the non-degenerate symmetric bilinear form $(-,-)_{E \otimes G}$ given by $\left(e \otimes g, e^{\prime} \otimes g^{\prime}\right)_{E \otimes G}:=\left(e, e^{\prime}\right)_{E} \cdot\left(g, g^{\prime}\right)_{G}$. Let $\mathbf{O G}_{2 r s}(E \otimes G)$ be the orthogonal Grassmannian parameterizing isotropic subspaces of $E \otimes G$ of dimension 2 rs. We name the connected components of $\mathbf{O G r}_{2 r s}(E \otimes G)$ such that $\mathbf{O G r}_{2 r s}^{+}(E \otimes G) \ni E \otimes U$ for an $s$-dimensional isotropic subspace $U$ of $G$.

Let $\Lambda$ be a Young diagram of type $\leq(r, s)$. For full flags by isotropic subspaces

$$
E_{\bullet}: E \supset E_{r} \supset \ldots E_{1} \supset E_{0}=0 \text { and } G_{\bullet}: G \supset G_{s} \supset \ldots G_{1} \supset G_{0}=0 \text {, }
$$

we put

$$
\mu_{\Lambda}\left(E_{\bullet}, G_{\bullet}\right):=\sum_{i=0}^{r+s}\left(\mathbb{F}_{i}^{\Lambda}\left(E_{\bullet}\right)^{\perp} \otimes \mathbb{F}_{i}^{\Lambda^{*}}\left(G_{\bullet}\right)+\mathbb{F}_{i}^{\Lambda}\left(E_{\bullet}\right) \otimes \mathbb{F}_{i}^{\Lambda^{*}}\left(G_{\bullet}\right)^{\perp}\right) \subset E \otimes G,
$$

where we used Notation 2.2. You can easily check that $\mu_{\Lambda}\left(E_{\bullet}, G_{\bullet}\right)$ is a $2 r s$ dimensional isotropic subspace of $E \otimes G$. So associating $\mu_{\Lambda}\left(E_{\bullet}, G_{\bullet}\right)$ to $\left(E_{\bullet}, G_{\bullet}\right)$, we obtain a morphism

$$
\mu_{\Lambda}: \mathbf{F l}(E) \times \mathbf{F l}(G) \rightarrow \mathbf{O G r}_{2 r s}(E \otimes G) .
$$

Lemma 2.3 ([A], Lemma 3.2.1). We have $\operatorname{Im} \mu_{\Lambda} \subset \mathbf{O G r}_{2 r s}^{+}(E \otimes G)$ if $|\Lambda|$ is even, and $\operatorname{Im} \mu_{\Lambda} \subset \mathbf{O G r}_{2 r s}^{-}(E \otimes G)$ if $|\Lambda|$ is odd.

2.5. The moduli stack of parabolic symplectic bundles. In this section we shall define a moduli stack of parabolic symplectic bundles, and introduce notation for line bundles on the moduli stack. We shall work over an algebraically closed field $k$ of characteristic zero.

Let $C$ be a smooth projective curve of genus $g, p^{(1)}, \ldots, p^{(m)}$ be distinct smooth points of $C$, and $L$ a line bundle on $C$. Put $\vec{p}:=\left(p^{(1)}, \ldots, p^{(m)}\right)$.

Definition 2.4. We define the moduli stack $M_{2 r}(C, \vec{p} ; L)$ as follows. For an affine $k$-scheme $T$, an object of the groupoid $M_{2 r}(C, \vec{p} ; L)(T)$ is the following data:

- a locally free $\mathcal{O}_{C \times T}$-module $\mathcal{E}$ of rank $2 r$,

- a non-degenerate alternate bilinear form $\mathcal{E} \otimes \mathcal{E} \rightarrow p r_{C}^{*} L$,

- for every point $p^{(j)}(1 \leq j \leq m)$, a full flag of $\mathcal{E}^{(j)}:=\left.\mathcal{E}\right|_{p^{(j)} \times T}$ by isotropic subbundles

$$
\mathcal{E}_{\bullet}^{(j)}: \mathcal{E}^{(j)} \supset \mathcal{E}_{r}^{(j)} \supset \cdots \supset \mathcal{E}_{1}^{(j)} \supset \mathcal{E}_{0}^{(j)}=0
$$

Isomorphisms of the groupoid $M_{2 r}(C, \vec{p} ; L)(T)$ are defined obviously.

An object of $M_{2 r}(C, \vec{p} ; L)(T)$ is called a parabolic symplectic bundle with values in $L$ on $C$ parameterized by $T$, and an object of $M_{2 r}(C, \vec{p} ; L)(\operatorname{Spec} k)$ is simply called a parabolic symplectic bundle with values in $L$ on $C$. 
Let

$$
\left(\mathcal{E}^{\text {univ }}, \mathcal{E}^{\text {univ }} \otimes \mathcal{E}^{\text {univ }} \rightarrow p r_{C}^{*} L, \mathcal{E}_{\bullet}^{\text {univ }(j)}(1 \leq j \leq m)\right)
$$

be the universal object of the moduli stack $M_{2 r}(C, \vec{p} ; L)$.

Definition 2.5. Let $n$ be an integer. Let each point $p^{(j)}(1 \leq j \leq m)$ be given a tuple of integers $\Lambda^{(j)}=\left(\lambda_{1}^{(j)}, \ldots, \lambda_{r}^{(j)}\right)$, and put $\vec{\Lambda}:=\left(\Lambda^{(1)}, \ldots, \Lambda^{(m)}\right)$. We denote by $\Xi_{M_{2 r}(C, \vec{p} ; L)}^{(n ; \vec{\Lambda})}$, or simply $\Xi^{(n ; \vec{\Lambda})}$, the line bundle

$$
\left(\operatorname{det} \mathbb{R} p r_{*} \mathcal{E}^{\text {univ }}\right)^{\otimes(-n)} \otimes \bigotimes_{j=1}^{m} \bigotimes_{i=1}^{r}\left(\frac{\mathcal{E}_{i-1}^{u n i v(j) \perp}}{\mathcal{E}_{i}^{u n i v(j) \perp}}\right)^{\otimes \lambda_{i}^{(j)}}
$$

on $M_{2 r}(C, \vec{p} ; L)$, where $p r$ is the projection $C \times M_{2 r}(C, \vec{p} ; L) \rightarrow M_{2 r}(C, \vec{p} ; L)$.

For later use, we introduce notation for orthogonal bundles as well.

Definition 2.6. An orthogonal vector bundle with values in $L$ on $C$ is a vector bundle $F$ on $C$ together with a non-degenerate symmetric bilinear form $F \otimes F \rightarrow L$.

We denote by $N_{2 t}(C ; L)$ the moduli stack of rank $2 t$ orthogonal vector bundles with values in $L$ on $C$.

Consider the special case where $L=\omega_{C}$. The moduli stack $N_{2 t}\left(C ; \omega_{C}\right)$ is a disjoint union of the open and closed substacks $N_{2 t}^{+}\left(C ; \omega_{C}\right)$ and $N_{2 t}^{-}\left(C ; \omega_{C}\right)$. Here an orthogonal vector bundle $F$ with values in $\omega_{C}$ lies in the component $N_{2 t}^{+}\left(C ; \omega_{C}\right)$ if and only if $\operatorname{dim} \mathrm{H}^{0}(C, F)$ is even.

If $\mathcal{F}^{\text {univ }}$ is the universal orthogonal vector bundle on $C \times N_{2 t}\left(C ; \omega_{C}\right)$, then the line bundle $\mathcal{D}:=\left(\operatorname{det} \mathbb{R} p r_{*} \mathcal{F}^{\text {univ }}\right)^{\vee}$ on $N_{2 t}\left(C ; \omega_{C}\right)$ is called the determinant bundle, where $p r: C \times N_{2 t}\left(C ; \omega_{C}\right) \rightarrow N_{2 t}\left(C ; \omega_{C}\right)$ is the projection. The determinant line bundle $\mathcal{D}$ has a canonical square root $\mathcal{P}$, the pfaffian bundle (cf. [L-S, Proposition 7.9]). Moreover the pfaffian bundle $\mathcal{P}$ has a canonical section $\Theta$ called the pfaffian divisor whose square $\Theta^{\otimes 2}$ is the canonical section of the determinant bundle (cf. [L-S, Section 7.10]).

2.6. Strange duality for parabolic symplectic bundles. In this section we recall from $[\mathrm{A}]$ the formulation of the strange duality for parabolic symplectic bundles.

Let $C$ and $p^{(1)}, \ldots, p^{(m)}$ be as in Section 2.5. Assume that each point $p^{(j)}$ $(1 \leq j \leq m)$ is given a Young diagram $\Lambda^{(j)}$ of type $\leq(r, s)$.

For a rank $2 r$ parabolic symplectic bundle

$$
\mathbb{E}:=\left(E, E \otimes E \rightarrow \mathcal{O}_{C}, E_{\bullet}^{(j)}: E^{(j)} \supset E_{r}^{(j)} \supset \cdots \supset E_{1}^{(j)} \supset E_{0}^{(j)}=0(1 \leq j \leq m)\right)
$$

and a rank $2 s$ parabolic symplectic bundle

$\mathbb{G}:=\left(G, G \otimes G \rightarrow \omega_{C}(\vec{p}), G_{\bullet}^{(j)}: G^{(j)} \supset G_{s}^{(j)} \supset \cdots \supset G_{1}^{(j)} \supset G_{0}^{(j)}=0(1 \leq j \leq m)\right)$, let $K$ be the kernel of the morphism

$$
E \otimes G \rightarrow \bigoplus_{j=1}^{m} \frac{E^{(j)} \otimes G^{(j)}}{\mu_{\Lambda^{(j)}}\left(E_{\bullet}^{(j)}, G_{\bullet}^{(j)}\right)},
$$

where the vector space $\left(E^{(j)} \otimes G^{(j)}\right) / \mu_{\Lambda^{(j)}}\left(E_{\bullet}^{(j)}, G_{\bullet}^{(j)}\right)$ is considered to be a skyscraper sheaf at $p^{(j)}$. (Recall that $E^{(j)}:=\left.E\right|_{p^{(j)}}$ and $G^{(j)}:=\left.G\right|_{p^{(j)}}$, and see Section 2.4 for the definition of $\mu_{\Lambda}$.)

The alternate bilinear forms of $E$ and $G$ determine a symmetric bilinear form $(E \otimes G) \otimes(E \otimes G) \rightarrow \omega(\vec{p})$ of $E \otimes G$. You can check easily that the restriction to $K$ of this symmetric bilinear form gives rise to a symmetric bilinear form $K \otimes K \rightarrow \omega_{C}$. 
Since $\operatorname{deg} K=4 r s(g-1)$, it is non-degenerate. Thus $K$ is an orthogonal bundle with values in $\omega_{C}$ on $C$. We define the morphism

$$
\tau_{(C ; \vec{\Lambda})}: M_{2 r}\left(C, \vec{p} ; \mathcal{O}_{C}\right) \times M_{2 s}\left(C, \vec{p} ; \omega_{C}(\vec{p})\right) \rightarrow N_{4 r s}\left(C ; \omega_{C}\right)
$$

by $(\mathbb{E}, \mathbb{G}) \mapsto K$. We have the following lemmas.

Lemma 2.7 ([A], Lemma 4.2.1). If $\sum_{j=1}^{m}\left|\Lambda^{(j)}\right|$ is even, then $\operatorname{Im} \tau_{(C ; \vec{\Lambda})} \subset N_{4 r s}^{+}\left(C ; \omega_{C}\right)$. If $\sum_{j=1}^{m}\left|\Lambda^{(j)}\right|$ is odd, then $\operatorname{Im} \tau_{(C ; \vec{\Lambda})} \subset N_{4 r s}^{-}\left(C ; \omega_{C}\right)$.

Lemma 2.8 ([A], Lemma 4.2.2). Let $\mathcal{P}$ be the pfaffian bundle on $N_{4 r s}\left(C ; \omega_{C}\right)$. Then we have an isomorphism

$$
\tau_{(C ; \vec{\Lambda})}^{*} \mathcal{P} \simeq \Xi_{M_{2 r}\left(C, \vec{p} ; \mathcal{O}_{C}\right)}^{(s ; \vec{A})} \otimes \Xi_{M_{2 s}\left(C, \vec{p} ; \omega_{C}(\vec{p})\right)}^{\left(r ; \vec{\Lambda}^{*}\right)}
$$

of line bundles on $M_{2 r}\left(C, \vec{p} ; \mathcal{O}_{C}\right) \times M_{2 s}\left(C, \vec{p} ; \omega_{C}(\vec{p})\right)$, where $\vec{\Lambda}^{*}=\left(\Lambda^{(1) *}, \ldots, \Lambda^{(m) *}\right)$.

If $\Theta$ is the canonical section of the pfaffian bundle $\mathcal{P}$ (cf. Section 2.5), then $\tau_{(C ; \vec{\Lambda})}^{*} \Theta$ induces the duality map

$\mathrm{H}^{0}\left(M_{2 r}\left(C, \vec{p} ; \mathcal{O}_{C}\right), \Xi_{M_{2 r}\left(C, \vec{p} ; \mathcal{O}_{C}\right)}^{(s ; \vec{\Lambda})}\right)^{*} \rightarrow \mathrm{H}^{0}\left(M_{2 s}\left(C, \vec{p} ; \omega_{C}(\vec{p})\right), \Xi_{M_{2 s}\left(C, \vec{p} ; \omega_{C}(\vec{p})\right)}^{\left(r ; \vec{\Lambda}^{*}\right)}\right)$

of vector spaces of global sections. The source and the target have the same dimension (cf. [A, §6]).

The following is the strange duality for parabolic symplectic bundles.

Conjecture 2.1. The morphism (2.4) is an isomorphism.

Remark 2.9. The $(-1)$-multiplication is an automorphism of parabolic symplectic bundles. It induces the multiplication by $(-1)^{\sum\left|\Lambda^{(j)}\right|}$ on the fibers of the line bundles $\Xi_{M_{2 r}\left(C, \vec{p} ; \mathcal{O}_{C}\right)}^{(s ; \vec{\Lambda})}$ and $\Xi_{M_{2 s}\left(C, \vec{p} ; \omega_{C}(\vec{p})\right)}^{\left(r ; \vec{\Lambda}^{*}\right)}$. Thus if $\sum_{j=1}^{m}\left|\Lambda^{(j)}\right|$ is odd, then the vector spaces $\mathrm{H}^{0}\left(M_{2 r}\left(C, \vec{p} ; \mathcal{O}_{C}\right), \Xi^{(s ; \vec{\Lambda})}\right)$ and $\mathrm{H}^{0}\left(M_{2 s}\left(C, \vec{p} ; \omega_{C}(\vec{p})\right), \Xi^{\left(r ; \vec{\Lambda}^{*}\right)}\right)$ are zero. So the conjecture is trivially true.

Remark 2.10. Take a smooth point $p^{(m+1)} \in C \backslash \vec{p}$, and label it by the empty Young diagram $\emptyset=(0 \geq \cdots \geq 0)$. Put $\overrightarrow{p^{\prime}}=\vec{p} \cup\left\{p^{(m+1)}\right\}$ and $\overrightarrow{\Lambda^{\prime}}=\vec{\Lambda} \cup\{\emptyset\}$. By associating to an object

$$
\mathbb{G}=\left(G, G \otimes G \rightarrow \omega_{C}\left(\overrightarrow{p^{\prime}}\right), G_{\bullet}^{(j)}(1 \leq j \leq m+1)\right) \in M_{2 s}\left(C, \overrightarrow{p^{\prime}} ; \omega_{C}\left(\overrightarrow{p^{\prime}}\right)\right)
$$

the object

$$
\left(G^{\prime}, G^{\prime} \otimes G^{\prime} \rightarrow \omega_{C}(\vec{p}), G_{\bullet}^{(j)}(1 \leq j \leq m)\right) \in M_{2 s}\left(C, \vec{p} ; \omega_{C}(\vec{p})\right)
$$

where $G^{\prime}:=\operatorname{Ker}\left(\left.G \rightarrow G\right|_{p^{(m+1)}} / G_{s}^{(m+1)}\right)$, we have a morphism

$$
g: M_{2 s}\left(C, \overrightarrow{p^{\prime}} ; \omega_{C}\left(\overrightarrow{p^{\prime}}\right)\right) \rightarrow M_{2 s}\left(C, \vec{p} ; \omega_{C}(\vec{p})\right) .
$$

Let

$$
f: M_{2 r}\left(C, \overrightarrow{p^{\prime}} ; \mathcal{O}_{C}\right) \rightarrow M_{2 r}\left(C, \vec{p} ; \mathcal{O}_{C}\right)
$$

be the morphism that forgets the filtration at $p^{(m+1)}$. We have

$$
f^{*} \Xi^{(s ; \vec{\Lambda})} \simeq \Xi^{\left(s ; \vec{\Lambda}^{\prime}\right)} \text { and } g^{*} \Xi^{\left(r ; \vec{\Lambda}^{*}\right)} \simeq \Xi^{\left(r ;{\overrightarrow{\Lambda^{\prime}}}^{*}\right)}
$$


and the diagram

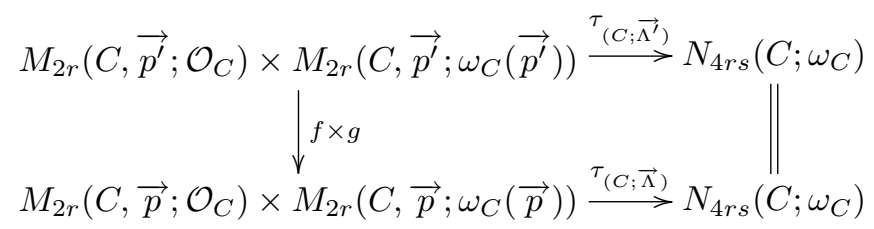

commutes. This induces a commutative diagram

$$
\begin{gathered}
H^{0}\left(M_{2 r}\left(C, \vec{p} ; \mathcal{O}_{C}\right), \Xi^{(s ; \vec{\Lambda})}\right)^{\vee} \longrightarrow H^{0}\left(M_{2 s}\left(C, \vec{p} ; \omega_{C}(\vec{p})\right), \Xi^{\left(r ; \vec{\Lambda}^{*}\right)}\right) \\
\left(f^{*}\right)^{\vee} \mid \simeq \quad g^{*} \downarrow \\
H^{0}\left(M_{2 r}\left(C, \overrightarrow{p^{\prime}} ; \mathcal{O}_{C}\right), \Xi^{\left(s ; \overrightarrow{\Lambda^{\prime}}\right)}\right)^{\vee} \longrightarrow H^{0}\left(M_{2 s}\left(C, \overrightarrow{p^{\prime}} ; \omega_{C}\left(\overrightarrow{p^{\prime}}\right)\right), \Xi^{\left(r ; \overrightarrow{\Lambda^{\prime *}}\right)}\right),
\end{gathered}
$$

where the horizontal arrows are strange duality maps. Here $f^{*}$ and $g^{*}$ are isomorphisms since $f$ and $g$ are flag-variety bundles.

By this, in order to see whether the strange duality map is an isomorphism or not, we may add an extra point and label it by the empty Young diagram.

\section{Conformal Blocks}

In this section we recall basic facts about conformal blocks. Our references are [B96], [S], [TUY].

We use the following usual notations for Lie algebras.

- $\mathfrak{g}$ is a simple Lie algebra, and $\mathfrak{h}$ is a fixed Cartan subalgebra.

- $G$ and $T$ are the corresponding simple, simply-connected Lie group and its maximal torus.

- $\mathfrak{h}^{*} \supset R(\mathfrak{g}, \mathfrak{h})$ is the root system, and we fix a basis $\left\{\alpha_{1}, \ldots, \alpha_{n}\right\}$.

- $\mathfrak{h}^{*} \supset P$ is the weight lattice, and $P_{+}$is the set of dominant weights.

- For $\lambda \in P_{+}, V_{\lambda}$ denotes the finite-dimensional irreducible $\mathfrak{g}$-module with highest weight $\lambda$. A highest vector, unique up to scalar, is denoted by $v_{\lambda}$.

- $(-,-)$ is the normalized Killing form (i.e. $\left(H_{\beta}, H_{\beta}\right)=2$ for long roots $\left.\beta\right)$. By this we identify $\mathfrak{h}$ and $\mathfrak{h}^{*}$.

- $\theta$ is the highest root of $R(\mathfrak{g}, \mathfrak{h})$, and $\rho$ is the half-sum of the positive roots.

- For $l \in \mathbb{N}, P_{l}:=\left\{\lambda \in P_{+} \mid \lambda\left(H_{\theta}\right) \leq l\right\}$.

- $g^{*}:=(\rho, \theta)+1$.

3.1. Definition of conformal blocks. Let $\widehat{\mathfrak{g}}$ be the affine Lie algebra

$$
\widehat{\mathfrak{g}}:=\mathfrak{g} \otimes \mathbb{C}((z)) \oplus \mathbb{C} c,
$$

where $c$ is a center and the bracket is given by

$$
[X \otimes f, Y \otimes g]:=[X, Y] \otimes f g+(X \mid Y) \operatorname{Res}(g d f) \cdot c .
$$

Put $\widehat{\mathfrak{g}}_{+}:=\mathfrak{g} \otimes z \mathbb{C}[[z]], \widehat{\mathfrak{g}}_{-}:=\mathfrak{g} \otimes z^{-1} \mathbb{C}\left[z^{-1}\right]$ and $\mathfrak{p}:=\mathfrak{g} \oplus \mathbb{C} c \oplus \widehat{\mathfrak{g}}_{+}$.

To each $\lambda \in P_{l}$, we can associate an integrable $\widehat{\mathfrak{g}}$-module $\mathcal{H}^{\widehat{\mathfrak{g}}}(\lambda ; l)$ of level $l$, which is characterized by the property:

The subspace annihilated by $\widehat{\mathfrak{g}}_{+}$is isomorphic to $V_{\lambda}$ as a $\mathfrak{g}$-module.

We sometimes simply write $\mathcal{H}(\lambda)$ for $\mathcal{H}^{\widehat{\mathfrak{g}}}(\lambda ; l)$ when $\widehat{\mathfrak{g}}$ and $l$ are clear from the context. The construction of $\mathcal{H}(\lambda)$ is as follows. By letting $\widehat{\mathfrak{g}}_{+}$act trivially on $V_{\lambda}$, and $c$ by $l \cdot \operatorname{Id}_{V_{\lambda}}, V_{\lambda}$ becomes a p-module. Put $\mathcal{V}(\lambda):=U(\widehat{\mathfrak{g}}) \otimes_{U(\mathfrak{p})} V_{\lambda}$. Let $\mathcal{Z}(\lambda) \subset \mathcal{V}(\lambda)$ be the $\widehat{\mathfrak{g}}$-submodule generated by $\left(X_{\theta} \otimes z^{-1}\right)^{l-\lambda\left(H_{\theta}\right)+1} v_{\lambda}$. Then $\mathcal{H}(\lambda):=\mathcal{V}(\lambda) / \mathcal{Z}(\lambda)$. We identify $V_{\lambda}$ with the subspace $1 \otimes V_{\lambda} \subset \mathcal{H}(\lambda)$. 
Let $C$ be a proper connected nodal algebraic curve over $\mathbb{C}$, and $p^{(1)}, \ldots, p^{(m)}$ smooth points of $C$. We assume that each irreducible component of $C$ contains at least one of $p^{(i)}$. Put $U:=C \backslash\left\{p^{(1)}, \ldots, p^{(m)}\right\}$. Note that by the assumption, $U$ is affine. We fix a formal parameter $\widehat{\mathcal{O}}_{C, p^{(i)}} \simeq \mathbb{C}\left[\left[z_{i}\right]\right]$ at $p^{(i)}$. For $f \in \mathcal{O}(U), f_{p^{(i)}} \in$ $\mathbb{C}\left[\left[z_{i}\right]\right]$ denotes the Laurent expansion of $f$ at $p^{(i)}$. For $\vec{\lambda}=\left(\lambda^{(1)}, \ldots, \lambda^{(m)}\right) \in P_{l}^{\oplus m}$, put $\mathcal{H}(\vec{\lambda}):=\mathcal{H}\left(\lambda^{(1)}\right) \otimes \cdots \otimes \mathcal{H}\left(\lambda^{(m)}\right)$. The Lie algebra $\mathfrak{g} \otimes \mathcal{O}(U)$ acts on $\mathcal{H}(\vec{\lambda})$ by

$$
(X \otimes f) \cdot\left(v_{1} \otimes \cdots \otimes v_{m}\right):=\sum_{1 \leq i \leq m} v_{1} \otimes \cdots \otimes\left(X \otimes f_{p^{(i)}}\right) v_{i} \otimes \cdots \otimes v_{m} .
$$

The dual conformal block $V_{C}^{\mathfrak{g}}(\vec{p} ; \vec{\lambda})$ is defined to be $\mathcal{H}_{\vec{\lambda}} /(\mathfrak{g} \otimes \mathcal{O}(U)) \mathcal{H}_{\vec{\lambda}}$, and the conformal block $V_{C}^{\mathfrak{g} \dagger}(\vec{p} ; \vec{\lambda})$ with level $l$ is defined to be the dual vector space of $V_{C}^{\mathfrak{g}}(\vec{p} ; \vec{\lambda})$. When $\mathfrak{g}$ is clear from the context, we drop $\mathfrak{g}$ from the notation and simply write $V_{C}(\vec{p} ; \vec{\lambda})$ and $V_{C}^{\dagger}(\vec{p} ; \vec{\lambda})$.

In the definition of conformal blocks, we used infinite-dimensional representations $\mathcal{H}_{\lambda}$ of $\widehat{\mathfrak{g}}$. We can replace some of them by finite-dimensional representations of $\mathfrak{g}$ as follows. Assume that each irreducible component of $C$ contains at least one of $p^{(2)}, \ldots, p^{(m)}$. Put $U^{\prime}:=C \backslash\left\{p^{(2)}, \ldots, p^{(m)}\right\}$ and $\overrightarrow{\lambda^{\prime}}:=\left(\lambda^{(2)}, \ldots, \lambda^{(m)}\right)$. The Lie algebra $\mathfrak{g} \otimes \mathcal{O}\left(U^{\prime}\right)$ acts on $V_{\lambda^{(1)}} \otimes \mathcal{H}\left(\overrightarrow{\lambda^{\prime}}\right)$ by

$$
\begin{aligned}
(X \otimes f) \cdot\left(v_{1} \otimes \cdots \otimes v_{m}\right):= & f\left(p^{(1)}\right)\left(X v_{1}\right) \otimes v_{2} \otimes \cdots \otimes v_{m} \\
& +\sum_{2 \leq i \leq m} v_{1} \otimes \cdots \otimes\left(X \otimes f_{p^{(i)}}\right) v_{i} \otimes \cdots \otimes v_{m} .
\end{aligned}
$$

Since the subspace $V_{\lambda^{(1)}} \subset \mathcal{H}\left(\lambda^{(1)}\right)$ is annihilated by $\widehat{\mathfrak{g}}_{+}$, this action is the restriction to the Lie subalgebra $\mathfrak{g} \otimes \mathcal{O}\left(U^{\prime}\right)$ of the action of $\mathfrak{g} \otimes \mathcal{O}(U)$ on $\mathcal{H}(\vec{\lambda})=\mathcal{H}\left(\lambda^{(1)}\right) \otimes$ $\mathcal{H}\left(\overrightarrow{\lambda^{\prime}}\right)$. So we have the morphism

$V_{C}(\vec{P} ; \vec{\lambda})=\mathcal{H}(\vec{\lambda}) /(\mathfrak{g} \otimes \mathcal{O}(U)) \mathcal{H}(\vec{\lambda}) \rightarrow\left(V_{\lambda^{(1)}} \otimes \mathcal{H}\left(\overrightarrow{\lambda^{\prime}}\right)\right) /\left(\mathfrak{g} \otimes \mathcal{O}\left(U^{\prime}\right)\right)\left(V_{\lambda^{(1)}} \otimes \mathcal{H}\left(\overrightarrow{\lambda^{\prime}}\right)\right)$

By [B96, Proposition 2.3] and [S, Proposition (2.3.4)], this morphism is an isomorphism.

3.2. Factorization theorem. Assume that $C$ is a union of connected nodal curves $C_{1}$ and $C_{2}$, and that $C_{1}$ and $C_{2}$ intersect at only one point $u$, which is a node. Let $p^{(1)}, \ldots, p^{(m)}$ be smooth points of $C_{1}$, and $q^{(1)}, \ldots, q^{(n)}$ smooth points of $C_{2}$. We assume that each irreducible component contains at least one marked point. Put $U:=C \backslash\left\{p^{(1)}, \ldots, p^{(m)}, q^{(1)}, \ldots, q^{(n)}\right\}, U_{1}:=C_{1} \backslash\left\{p^{(1)}, \ldots, p^{(m)}\right\}$ and $U_{2}:=$ $C_{2} \backslash\left\{q^{(1)}, \ldots, q^{(n)}\right\}$. Let

$$
\mathfrak{n}: \widetilde{C}:=C_{1} \sqcup C_{2} \rightarrow C=C_{1} \cup C_{2}
$$

be the partial normalization at $u$. Put $\left\{u_{1}, u_{2}\right\}:=\mathfrak{n}^{-1}(u)$ with $u_{i} \in C_{i}$.

Fix $\vec{\lambda} \in P_{l}^{\oplus m}$ and $\vec{\mu} \in P_{l}^{\oplus n}$. For $\nu \in P_{+}$, let $\gamma_{\nu} \in V_{\nu} \otimes V_{\nu^{*}}$ be a non-zero element annihilated by $\mathfrak{g}$, uniquely determined up to scalar. Consider the injection induced by $\gamma_{\nu}$

$$
\mathcal{H}_{\vec{\lambda}} \otimes \mathcal{H}_{\vec{\mu}} \hookrightarrow \mathcal{H}_{\vec{\lambda}} \otimes V_{\nu} \otimes V_{\nu^{*}} \otimes \mathcal{H}_{\vec{\mu}}
$$

Here the vector spaces $V_{\nu}$ and $V_{\nu^{*}}$ are regarded as associated to the points $u_{1}$ and $u_{2}$ respectively.

As in (3.2), $\mathcal{H}_{\vec{\lambda}} \otimes V_{\nu}$ and $V_{\nu^{*}} \otimes \mathcal{H}_{\vec{\mu}}$ have the action of the Lie algebras $\mathfrak{g} \otimes \mathcal{O}_{C_{1}}\left(U_{1}\right)$ and $\mathfrak{g} \otimes \mathcal{O}_{C_{2}}\left(U_{2}\right)$. As in $(3.1), \mathcal{H}_{\vec{\lambda}} \otimes \mathcal{H}_{\vec{\mu}}$ has the action of $\mathfrak{g} \otimes \mathcal{O}_{C}(U)$. We have a canonical injective map

$$
\mathfrak{g} \otimes \mathcal{O}_{C}(U) \hookrightarrow\left(\mathfrak{g} \otimes \mathcal{O}_{C_{1}}\left(U_{1}\right)\right) \oplus\left(\mathfrak{g} \otimes \mathcal{O}_{C_{2}}\left(U_{2}\right)\right)
$$


and the injection (3.3) is compatible with the actions of the Lie algebras.

So we have a natural morphism

$$
V_{C}(\vec{p} \cup \vec{q} ; \vec{\lambda} \cup \vec{\mu}) \rightarrow V_{C_{1}}\left(\vec{p} \cup\left\{u_{1}\right\} ; \vec{\lambda} \cup\{\nu\}\right) \otimes V_{C_{2}}\left(\vec{q} \cup\left\{u_{2}\right\} ; \vec{\mu} \cup\left\{\nu^{*}\right\}\right) .
$$

The following theorem is call the factorization theorem.

Theorem 3.1 ([TUY]Proposition 2.2.6). The morphism

$$
V_{C}(\vec{p} \cup \vec{q} ; \vec{\lambda} \cup \vec{\mu}) \rightarrow \bigoplus_{\nu \in P_{l}} V_{C_{1}}\left(\vec{p} \cup\left\{u_{1}\right\} ; \vec{\lambda} \cup\{\nu\}\right) \otimes V_{C_{2}}\left(\vec{q} \cup\left\{u_{2}\right\} ; \vec{\mu} \cup\left\{\nu^{*}\right\}\right)
$$

is an isomorphism.

3.3. Dimension of conformal blocks. In this section we calculate the dimensions of some conformal blocks on a pointed $\mathbb{P}^{1}$.

Let $\mathfrak{s} \simeq \mathfrak{s l}_{2}$ be the Lie subalgebra generated by $H_{\theta}, \mathfrak{g}_{\theta}$ and $\mathfrak{g}_{-\theta}$. A $\mathfrak{g}$-module $V$ decomposes as $V=\oplus_{i} V^{(i)}$ with $V^{(i)}$ a direct sum of $\mathfrak{s l}_{2}$-modules isomorphic to $S^{i} \mathbb{C}^{2}$.

The following description of conformal blocks on a 3 -pointed $\mathbb{P}^{1}$ is important.

Proposition 3.2 ([B96] Proposition 4.3). Fix 3 points $a, b, c$ on $\mathbb{P}^{1}$, and $\lambda, \mu, \nu \in$ $P_{l}$. The conformal block $V_{\mathbb{P}^{1}}^{\dagger}(a, b, c ; \lambda, \mu, \nu)$ is canonically isomorphic to the space of $\mathfrak{g}$-equivariant linear maps $\varphi: V_{\lambda} \otimes V_{\mu} \rightarrow V_{\nu}^{\vee}$ such that the composite

$$
V_{\lambda}^{(p)} \otimes V_{\mu}^{(q)} \rightarrow V_{\lambda} \otimes V_{\mu} \rightarrow V_{\nu}^{\vee} \rightarrow\left(V_{\nu}^{(r)}\right)^{\vee}
$$

is zero if $p+q+r>2 l$.

3.3.1. The case $\mathfrak{g}=\mathfrak{s o}_{2 N}$ with level one. Let $V$ be a $2 N$-dimensional vector space with a non-degenerate symmetric bilinear form $Q$. The Lie algebra $\mathfrak{s o}(V)$ is

$$
\mathfrak{s o}(V):=\{f: V \rightarrow V \mid Q(f(v), w)+Q(v, f(w))=0\} .
$$

If we choose a basis $\left\{\mathbf{e}_{1}, \ldots, \mathbf{e}_{2 N}\right\}$ of $V$ such that $Q\left(\mathbf{e}_{i}, \mathbf{e}_{j}\right)=Q\left(\mathbf{e}_{N+i}, \mathbf{e}_{N+j}\right)=0$ and $Q\left(\mathbf{e}_{i}, \mathbf{e}_{N+j}\right)=\delta_{i j}$ for $1 \leq i, j \leq N$, then the Lie algebra $\mathfrak{s o}(V)$ is identified with

$$
\mathfrak{s o}_{2 N}:=\left\{\left.X \in \operatorname{Mat}_{2 N \times 2 N}\right|^{t} X M_{2 N}+M_{2 N} X=0\right\},
$$

where

$$
M_{2 N}=\left(\begin{array}{c|c}
0 & I_{N} \\
\hline I_{N} & 0
\end{array}\right)
$$

Let $\mathfrak{h} \subset \mathfrak{s o}_{2 N}$ be the diagonal Cartan subalgebra. Put $H_{i}:=E_{i, i}-E_{N+i, N+i}$, and let $\left\{L_{i}\right\} \subset \mathfrak{h}^{*}$ be the dual basis, i.e. $\left\langle L_{i}, H_{j}\right\rangle=\delta_{i j}$. The roots of $\mathfrak{s o}_{2 N}$ are $\left\{ \pm L_{i} \pm L_{j}\right\}_{i<j}$. Take $R^{+}:=\left\{L_{i} \pm L_{j}\right\}_{i<j}$ as the positive roots. Then the root basis is

$$
L_{1}-L_{2}, L_{2}-L_{3}, \ldots, L_{N-1}-L_{N}, L_{N-1}+L_{N} .
$$

The highest $\operatorname{root} \theta$ is $L_{1}+L_{2}$. The weight lattice $P$ is

$$
\left\{\left(a_{1} L_{1}+\ldots a_{N} L_{N}\right) / 2 \mid a_{i} \in \mathbb{Z} \text { and } a_{i} \equiv a_{j}(\bmod 2)\right\} .
$$

Put $\alpha=\left(L_{1}+\cdots+L_{N-1}+L_{N}\right) / 2$ and $\beta=\left(L_{1}+\cdots+L_{N-1}-L_{N}\right) / 2$. Then we have

$$
P_{1}=\left\{0, L_{1}, \alpha, \beta\right\} .
$$

The representation $V_{L_{1}}$ is the standard representation $\mathbb{C}^{2 N}$, and the representations $V_{\alpha}, V_{\beta}$ are called half-spin representations (cf. [FH, §20]). 
Proposition 3.3. Take three points $a, b, c$ on $\mathbb{P}^{1}$ and weights $\lambda, \mu, \nu \in\left\{L_{1}, \alpha, \beta\right\}$. Then for the conformal block $V_{\mathbb{P}^{1}}^{\mathfrak{s o}_{2} \dagger}(a, b, c ; \lambda, \mu, \nu)$ of level one, if $N$ is even

$$
\operatorname{dim} V_{\mathbb{P}^{1}}^{\mathfrak{s o}_{2 N} \dagger}(a, b, c ; \lambda, \mu, \nu)=\left\{\begin{array}{ll}
1 & \text { if }\{\lambda, \mu, \nu\}=\left\{L_{1}, \alpha, \beta\right\} \\
0 & \text { otherwise }
\end{array},\right.
$$

and if $N$ is odd

$$
\operatorname{dim} V_{\mathbb{P}^{1}}^{\mathfrak{s o}_{2 N} \dagger}(a, b, c ; \lambda, \mu, \nu)=\left\{\begin{array}{ll}
1 & \text { if }\{\lambda, \mu, \nu\}=\left\{L_{1}, \alpha, \alpha\right\} \text { or }\left\{L_{1}, \beta, \beta\right\} \\
0 & \text { otherwise }
\end{array} .\right.
$$

Proof. We give the proof only for the case $N$ even. The irreducible decompositions of the tensor products of the $\mathfrak{s o}_{2 N}$-modules $V_{L_{1}}, V_{\alpha}$ and $V_{\beta}$ are as follows:

$$
\begin{aligned}
& V_{L_{1}} \otimes V_{L_{1}} \simeq V_{L_{1}+L_{2}} \oplus V_{2 L_{1}} \oplus V_{0} \\
& V_{L_{1}} \otimes V_{\alpha} \simeq V_{L_{1}+\alpha} \oplus V_{\beta}, \quad V_{L_{1}} \otimes V_{\beta} \simeq V_{L_{1}+\beta} \oplus V_{\alpha} \\
& V_{\alpha} \otimes V_{\alpha} \simeq V_{2 \alpha} \oplus \oplus_{i=1}^{N / 2} V_{L_{1}+\cdots+L_{N-2 i}}, \quad V_{\beta} \otimes V_{\beta} \simeq V_{2 \beta} \oplus \oplus_{i=1}^{N / 2} V_{L_{1}+\cdots+L_{N-2 i}} \\
& V_{\alpha} \otimes V_{\beta} \simeq \oplus_{i=1}^{N / 2-1} V_{L_{1}+\cdots+L_{N-2 i+1}} .
\end{aligned}
$$

This and the fact that $V_{\alpha}^{\vee} \simeq V_{\alpha}$ and $V_{\beta}^{\vee} \simeq V_{\beta}$ for $N$ even ([FH, Exercise 19.5]) imply that $\operatorname{dim} V_{\mathbb{P 1}}^{\mathfrak{s} \mathfrak{o}_{2 N} \dagger}(a, b, c ; \lambda, \mu, \nu)=0$ unless $\{\lambda, \mu, \nu\}=\left\{L_{1}, \alpha, \beta\right\}$. In the case $\{\lambda, \mu, \nu\}=\left\{L_{1}, \alpha, \beta\right\}$, the composite

$$
V_{L_{1}}^{(1)} \otimes V_{\alpha}^{(1)} \rightarrow V_{L_{1}} \otimes V_{\alpha} \rightarrow V_{\beta}^{\vee} \rightarrow\left(V_{\beta}^{(1)}\right)^{\vee}
$$

is zero since $\mathbb{C}^{2} \otimes \mathbb{C}^{2} \simeq S^{2} \mathbb{C}^{2} \oplus \mathbb{C}$ as $\mathfrak{s l}_{2}$-modules. Hence $\operatorname{dim} V_{\mathbb{P}^{1}}^{\mathfrak{s o}_{2 N} \dagger}\left(a, b, c ; L_{1}, \alpha, \beta\right)=$ 1.

The proof of the odd case, which is not used in this paper, is left to the reader.

Corollary 3.4. Assume that $N$ is even. Take points $p^{(1)}, \ldots, p^{(2 a+2 b)}$ on $\mathbb{P}^{1}$. Then we have

$$
\operatorname{dim} V_{\mathbb{P}^{1}}^{\mathfrak{s o}_{2 N} \dagger}\left(p^{(1)}, \ldots, p^{(2 a+2 b)} ; \alpha^{2 a}, \beta^{2 b}\right)=1 .
$$

Proof. By induction on the number of points, this follows from the above proposition and the fact that the dimensions of conformal blocks obey the fusion rule (cf. $[\mathrm{B} 96$, Part II]).

3.3.2. The case $\mathfrak{g}=\mathfrak{s p}_{2 r}$. Let $V$ be a $2 r$-dimensional vector space with a nondegenerate alternate bilinear form $(-,-)$. The Lie algebra $\mathfrak{s p}(V)$ is

$$
\mathfrak{s p}(V):=\{f: V \rightarrow V \mid(f(v), w)+(v, f(w))=0\} .
$$

If we choose a basis $\left\{\mathbf{e}_{1}, \ldots, \mathbf{e}_{2 r}\right\}$ of $V$ such that $\left(\mathbf{e}_{i}, \mathbf{e}_{j}\right)=\left(\mathbf{e}_{r+i}, \mathbf{e}_{r+j}\right)=0$ and $\left(\mathbf{e}_{i}, \mathbf{e}_{r+j}\right)=\delta_{i j}$ for $1 \leq i, j \leq r$, then $\mathfrak{s p}(V)$ is identified with

$$
\mathfrak{s p}_{2 r}:=\left\{\left.X \in \operatorname{Mat}_{2 r \times 2 r}\right|^{t} X J_{2 r}+J_{2 r} X=0\right\},
$$

where

$$
J_{2 r}=\left(\begin{array}{c|c}
0 & I_{r} \\
\hline-I_{r} & 0
\end{array}\right)
$$

Let $\mathfrak{h} \subset \mathfrak{s p}_{2 r}$ be the diagonal Cartan subalgebra. Put $H_{i}:=E_{i, i}-E_{r+i, r+i}$, and let $\left\{L_{i}\right\} \subset \mathfrak{h}^{*}$ be the dual basis, i.e. $\left\langle L_{i}, H_{j}\right\rangle=\delta_{i j}$. The roots of $\mathfrak{s p}_{2 r}$ are $\left\{ \pm L_{i} \pm L_{j}\right\}_{i<j} \cup\left\{2 L_{i}\right\}_{1 \leq i \leq r}$ Take $R^{+}:=\left\{L_{i}+L_{j}\right\}_{i \leq j} \cup\left\{L_{i}-L_{j}\right\}_{i<j}$ as the positive roots. Then the root basis is

$$
L_{1}-L_{2}, L_{2}-L_{3}, \ldots, L_{r-1}-L_{r}, 2 L_{r} .
$$

The highest root $\theta$ is $2 L_{1}$. The weight lattice $P$ is $\left\{a_{1} L_{1}+\ldots a_{r} L_{r} \mid a_{i} \in \mathbb{Z}\right\}$. A weight $\lambda=a_{1} L_{1}+\ldots a_{r} L_{r}$ is dominant if and only if $a_{1} \geq \cdots \geq a_{r} \geq 0$. 
We have

$$
P_{l}=\left\{a_{1} L_{1}+\ldots a_{r} L_{r} \in P_{+} \mid a_{r} \leq l\right\} .
$$

So there is a one-to-one correspondence between $P_{l}$ and the set of all Young diagrams of type $\leq(r, l)$.

The representation $V_{L_{1}}$ is the standard representation $\mathbb{C}^{2 r}$. For $\vec{a}=\left(a_{1} \geq\right.$ $\left.\cdots \geq a_{r} \geq 0\right)$, the $\mathfrak{s p}_{2 r}$-module $\mathrm{H}^{0}(\mathbf{F l}, \mathcal{O}(\vec{a}))$ in Proposition 2.1 is isomorphic to $V_{a_{1} L_{1}+\cdots+a_{r} L_{r}}$. All the $\mathfrak{s p}_{2 r}$-modules are self-dual, i.e., $V_{\lambda}^{\vee} \simeq V_{\lambda}$.

Proposition 3.5. Fix the level l. Take three points $a, b, c$ on $\mathbb{P}^{1}$, and two weights $\lambda, \mu \in P_{l}$. Then the dimension of the conformal block $V_{\mathbb{P 1}}^{\mathfrak{s p}_{2 r}^{\dagger}}\left(a, b, c ; L_{1}, \lambda, \mu\right)$ of level $l$ is 1 if the Young diagram $\mu$ is obtained from $\lambda$ by adding or deleting one box, and 0 otherwise.

Proof. By [L], for $\lambda \in P_{+}$, we have

$$
V_{L_{1}} \otimes V_{\lambda} \simeq \sum_{\nu} V_{\nu}
$$

where $\nu$ runs through all Young diagrams that are obtained from $\lambda$ by adding or deleting one box. This proves the part " 0 otherwise" in the proposition. Assume that the Young diagram $\mu$ is obtained from $\lambda$ by adding or deleting one box. For a $\mathfrak{s p}_{2 r}$-equivariant linear map $\varphi: V_{L_{1}} \otimes V_{\lambda} \rightarrow V_{\mu}\left(\simeq V_{\mu}^{\vee}\right)$, the composite

$$
V_{L_{1}}^{(1)} \otimes V_{\lambda}^{(l)} \rightarrow V_{L_{1}} \otimes V_{\lambda} \rightarrow\left(V_{\mu}\right)^{\vee} \rightarrow\left(V_{\mu}^{(l)}\right)^{\vee}
$$

is zero since $\mathbb{C}^{2} \otimes S^{l} \mathbb{C}^{2}$ does not contain $S^{l} \mathbb{C}^{2}$ as an $\mathfrak{s l}_{2}$-submodule. Hence by Proposition 3.2, we have $\operatorname{dim} V_{\mathbb{P}^{1}}^{\mathfrak{s p}_{2 r}^{\dagger}}\left(a, b, c ; L_{1}, \lambda, \mu\right)=1$.

3.4. Rank-level duality of conformal blocks for $\left(\mathfrak{s p}_{2 r}, \mathfrak{s p}_{2 s}\right)$. In this section we define the rank-level duality map for conformal blocks of $\mathfrak{s p}_{2 r}$ and $\mathfrak{s p}_{2 s}$.

Let $W_{2 r}$ and $W_{2 s}$ be vector spaces of dimension $2 r$ and $2 s$ equipped with a nondegenerate alternate bilinear form. Put $N:=2 r s$. The tensor product $W_{2 N}:=$ $W_{2 r} \otimes W_{2 s}$ has the non-degenerate symmetric bilinear form determined by $(x \otimes$ $\left.y, x^{\prime} \otimes y^{\prime}\right)_{W_{2 N}}:=\left(x, x^{\prime}\right)_{W_{2 r}}\left(y, y^{\prime}\right)_{W_{2 s}}$.

Let $L: \mathfrak{s p}\left(W_{2 r}\right) \rightarrow \mathfrak{s o}\left(W_{2 r} \otimes W_{2 s}\right)$ and $R: \mathfrak{s p}\left(W_{2 s}\right) \rightarrow \mathfrak{s o}\left(W_{2 r} \otimes W_{2 s}\right)$ be the morphism of Lie algebras given by $\mathfrak{s p}\left(W_{2 r}\right) \ni \varphi \mapsto \varphi \otimes \operatorname{id}_{W_{2 s}} \in \mathfrak{s o}\left(W_{2 r} \otimes W_{2 s}\right)$ and $\mathfrak{s p}\left(W_{2 s}\right) \ni \psi \mapsto \operatorname{id}_{W_{2 r}} \otimes \psi \in \mathfrak{s o}\left(W_{2 r} \otimes W_{2 s}\right)$. We define the morphisms

$\left.\widehat{L}: \widehat{\mathfrak{s p}\left(W_{2 r}\right.}\right)\left(=\mathfrak{s p}\left(W_{2 r}\right) \otimes \mathbb{C}((z)) \oplus \mathbb{C} \cdot c\right) \rightarrow \mathfrak{s o ( W _ { 2 N } )}\left(=\mathfrak{s o}\left(W_{2 N}\right) \otimes \mathbb{C}((z)) \oplus \mathbb{C} \cdot c\right)$,

$\left.\widehat{R}: \widehat{\mathfrak{s p}\left(W_{2 s}\right)}\left(=\mathfrak{s p}\left(W_{2 s}\right) \otimes \mathbb{C}((z)) \oplus \mathbb{C} \cdot c\right) \rightarrow \mathfrak{s o} \widehat{\left(W_{2 N}\right.}\right)\left(=\mathfrak{s o}\left(W_{2 N}\right) \otimes \mathbb{C}((z)) \oplus \mathbb{C} \cdot c\right)$

by

$$
\begin{aligned}
& \widehat{L}(\varphi \otimes f(z)+a \cdot c)=L(\varphi) \otimes f(z)+s a \cdot c, \\
& \widehat{R}(\psi \otimes f(z)+a \cdot c)=R(\psi) \otimes f(z)+r a \cdot c .
\end{aligned}
$$

Then $\widehat{L}$ and $\widehat{R}$ are morphisms of Lie algebras.

Fix symplectic bases $\left\{\mathbf{e}_{1}, \ldots, \mathbf{e}_{2 r}\right\} \subset W_{2 r}$ and $\left\{\mathbf{g}_{1}, \ldots, \mathbf{g}_{2 s}\right\} \subset W_{2 s}$, i.e.,

$$
\begin{gathered}
\left(\mathbf{e}_{i}, \mathbf{e}_{j}\right)=\left(\mathbf{e}_{r+i}, \mathbf{e}_{r+j}\right)=0, \quad\left(\mathbf{e}_{i}, \mathbf{e}_{r+j}\right)=-\left(\mathbf{e}_{r+j}, \mathbf{e}_{i}\right)=\delta_{i j} \quad \text { for } 1 \leq i, j \leq r \\
\left(\mathbf{g}_{i}, \mathbf{g}_{j}\right)=\left(\mathbf{g}_{s+i}, \mathbf{g}_{s+j}\right)=0, \quad\left(\mathbf{g}_{i}, \mathbf{g}_{s+j}\right)=-\left(\mathbf{g}_{s+j}, \mathbf{g}_{i}\right)=\delta_{i j} \quad \text { for } 1 \leq i, j \leq s
\end{gathered}
$$

Let $\left\{\mathbf{f}_{l} \mid 1 \leq l \leq N\right\}$ be the set $\left\{\mathbf{e}_{i} \otimes \mathbf{g}_{j} \mid 1 \leq i \leq 2 r, 1 \leq j \leq s\right\}$. Determine $\mathbf{f}_{N+1}, \ldots, \mathbf{f}_{2 N}$ by the equalities

$$
\left(\mathbf{f}_{i}, \mathbf{f}_{N+j}\right)_{W_{2 N}}=\delta_{i j} \quad \text { and } \quad\left(\mathbf{f}_{N+i}, \mathbf{f}_{N+j}\right)_{W_{2 N}}=0 \quad \text { for } 1 \leq i, j \leq N .
$$

By these bases, we identify $\mathfrak{s p}\left(W_{2 r}\right), \mathfrak{s p}\left(W_{2 s}\right), \mathfrak{s o}\left(W_{2 N}\right)$ with $\mathfrak{s p}_{2 r}, \mathfrak{s p}_{2 s}, \mathfrak{s o}_{2 N}$ respectively. 
We identify $P_{s}$ of $\mathfrak{s p}_{2 r}$ with the set of Young diagrams of type $\leq(r, s)$, and $P_{r}$ of $\mathfrak{s p}_{2 s}$ with the set of Young diagram of type $\leq(s, r)$.

As in Section 3.3.1, we denote by $\alpha$ and $\beta$ the weights

$$
L_{1}+\cdots+L_{N-1}+L_{N} \text { and } L_{1}+\cdots+L_{N-1}-L_{N}
$$

of the Lie algebra $\mathfrak{s o}_{2 N}$ respectively.

By the morphism

$$
\widehat{L}+\widehat{R}: \widehat{\mathfrak{s p}_{2 r}} \oplus \widehat{\mathfrak{s p}_{2 s}} \rightarrow \widehat{\mathfrak{s o}_{2 N}}
$$

a $\widehat{\mathfrak{s o}_{2 N}}$-module can be regarded as a $\left(\widehat{\mathfrak{s p}_{2 r}} \oplus \widehat{\mathfrak{s p}_{2 s}}\right)$-module. If you regard the integrable $\widehat{\mathfrak{s o}_{2 N}}$-modules $\mathcal{H}^{\mathfrak{s o}_{2 N}}(\alpha ; 1)$ and $\mathcal{H}^{\mathfrak{s o}_{2 N}}(\beta ; 1)$ as $\left(\widehat{\mathfrak{s p}_{2 r}} \oplus \widehat{\mathfrak{s p}_{2 s}}\right)$-modules, then they decompose as follows:

Theorem 3.6 ([Has] Theorem4.2, Theorem 3.2). We have isomorphisms of $\left(\widehat{\mathfrak{s p}_{2 r}} \oplus\right.$ $\left.\widehat{\mathfrak{s p}_{2 s}}\right)$-modules

$$
\begin{aligned}
& \mathcal{H}^{\widehat{\mathfrak{s o}_{2 N}}}(\alpha ; 1) \simeq \bigoplus_{|\Lambda| \text { :even }} \mathcal{H}^{\widehat{\mathfrak{s p}_{2 r}}}(\Lambda ; s) \otimes \mathcal{H}^{\widehat{\mathfrak{p q}_{2 s}}}\left(\Lambda^{*} ; r\right)
\end{aligned}
$$

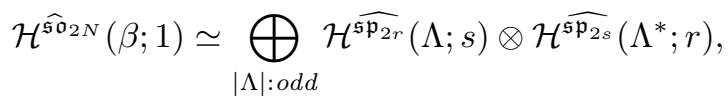

where $\Lambda$ runs through all Young diagrams of type $\leq(r, s)$ with $|\Lambda|$ even in (3.5), and with $|\Lambda|$ odd in (3.6).

Moreover, by restricting these isomorphisms to the subspaces annihilated by $\widehat{\mathfrak{s o}}_{2 N}+$, $\widehat{\mathfrak{s p}_{2 r}}, \widehat{\mathfrak{s p}_{2 s}}$, we have isomorphisms of $\mathfrak{s p}_{2 r} \oplus \mathfrak{s p}_{2 s}$-modules:

$$
\begin{aligned}
& V_{\alpha} \simeq \bigoplus_{|\Lambda|: \text { even }} V_{\Lambda} \otimes V_{\Lambda^{*}} \\
& V_{\beta} \simeq \bigoplus_{|\Lambda| \text { :odd }} V_{\Lambda} \otimes V_{\Lambda^{*}} .
\end{aligned}
$$

This theorem implies that there is a unique (up to scalar) non-zero morphism

$$
\widehat{\mathcal{H}^{\mathfrak{\mathfrak { s p } _ { 2 r }}}}(\Lambda ; s) \otimes \mathcal{H}^{\widehat{\mathfrak{s p}_{2 s}}}\left(\Lambda^{*} ; r\right) \rightarrow \widehat{\mathcal{H}^{\widehat{\mathfrak{s o}_{2 N}}}}\left((-1)^{|\Lambda|} ; 1\right),
$$

of $\widehat{\mathfrak{s p}_{2 r}} \oplus \widehat{\mathfrak{s p}_{2 s}}$-modules, where we understand that

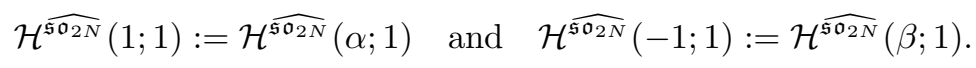

Fix smooth points $p^{(1)}, \ldots p^{(e)}$ on a projective nodal curve $C$ of arithmetic genus 0 , and Young diagrams $\Lambda^{(1)}, \ldots, \Lambda^{(e)}$ of type $\leq(r, s)$. Put $U:=C \backslash\left\{p^{(1)}, \ldots, p^{(e)}\right\}$, $\vec{p}:=\left(p^{(1)}, \ldots, p^{(e)}\right), \vec{\Lambda}:=\left(\Lambda^{(1)}, \ldots, \Lambda^{(e)}\right)$ and $\vec{\Lambda}^{*}:=\left(\Lambda^{(1) *}, \ldots, \Lambda^{(e) *}\right)$. Assume that $U$ is affine. Taking a tensor product of non-zero morphisms of $\widehat{\mathfrak{s p}_{2 r}} \oplus \widehat{\mathfrak{s p}_{2 s}}-$ modules

$$
\mathcal{H}^{\widehat{\mathfrak{s p}_{2 r}}}\left(\Lambda^{(i)} ; s\right) \otimes \mathcal{H}^{\widehat{\mathfrak{s p}_{2 s}}}\left(\Lambda^{(i) *} ; r\right) \rightarrow \widehat{\mathcal{H}^{\mathfrak{s o}_{2 N}}}\left((-1)^{\left|\Lambda^{(i)}\right|} ; 1\right)
$$

for $1 \leq i \leq e$, we have

$$
\left\{\bigotimes_{i=1}^{e} \widehat{\mathcal{H}^{\widehat{\mathfrak{p}_{2 r}}}}\left(\Lambda^{(i)} ; s\right)\right\} \otimes\left\{\bigotimes_{i=1}^{e} \widehat{\mathcal{H}^{\widehat{\mathfrak{s p}}}}\left(\Lambda^{(i) *} ; r\right)\right\} \rightarrow \bigotimes_{i=1}^{e} \widehat{\mathcal{H}^{\widehat{\mathfrak{s}_{2 N}}}}\left((-1)^{\mid \Lambda^{(i)}} ; 1\right)
$$

By (the tensor product of ) the formula (3.1), the Lie algebra $\left(\mathfrak{s p}_{2 r} \oplus \mathfrak{s p}_{2 s}\right) \otimes \mathcal{O}_{C}(U)$ acts on the source of (3.9), and the Lie algebra $\mathfrak{s o}_{2 N} \otimes \mathcal{O}_{C}(U)$ acts on the target of (3.9). The morphism (3.9) is compatible with these actions. Hence (3.9) induces a morphism

$$
V_{C}^{\mathfrak{s p}_{2 r}}(\vec{p} ; \vec{\Lambda}) \otimes V_{C}^{\mathfrak{s p}_{2 s}}\left(\vec{p} ; \vec{\Lambda}^{*}\right) \rightarrow V_{C}^{\mathfrak{s o}_{2 N}}(\vec{p} ; \vec{\epsilon}),
$$

where $\vec{\epsilon}:=\left((-1)^{\left|\Lambda^{(1)}\right|}, \ldots,(-1)^{\left|\Lambda^{(e)}\right|}\right)$. (Recall that here we understand that $+1=$ $\alpha$ and $-1=\beta$.) 
Taking the dual, we have

$$
V_{C}^{\mathfrak{s o}_{2 N}^{\dagger}}(\vec{p} ; \vec{\epsilon}) \rightarrow V_{C}^{\mathfrak{s p}_{2 r} \dagger}(\vec{p} ; \vec{\Lambda}) \otimes V_{C}^{\mathfrak{s p}_{2 s}^{\dagger}}\left(\vec{p} ; \vec{\Lambda}^{*}\right) .
$$

Now assume that both $e$ and $\sum_{j=1}^{e}\left|\Lambda^{(j)}\right|$ are even. Then by Corollary 3.4, we have

$$
\operatorname{dim} V_{C}^{\mathfrak{s o}_{2 N}^{\dagger}}(\vec{p} ; \vec{\epsilon})=1 .
$$

Hence the morphism (3.11) induces the morphism

$$
V_{C}^{\mathfrak{s p}_{2 r}^{\dagger}}(\vec{p} ; \vec{\Lambda})^{*} \rightarrow V_{C}^{\mathfrak{s p}_{2 s}^{\dagger}}\left(\vec{p} ; \vec{\Lambda}^{*}\right) .
$$

This map is called a rank-level duality map.

3.5. Compatibility of factorization and rank-level duality. In this section we show that the factorization isomorphism (3.4) is compatible with the rank-level duality map. This is not a new result. It is the commutative [NT, (2.14)]. In terms of generalized theta functions, the compatibility is also proved in [A].

Let $C=C_{1} \cup C_{2}$ be a projective nodal curve of arithmetic genus 0 with each $C_{i}$ connected, and $C_{1}$ and $C_{2}$ intersecting at only one point $u$ transversely. Let $p^{(1)}, \ldots, p^{(m)}$ be smooth points of $C_{1} \backslash\{u\}$, and $q^{(1)}, \ldots, q^{(n)}$ smooth points of $C_{2} \backslash\{u\}$. Let

$$
\mathfrak{n}: \widetilde{C}:=C_{1} \sqcup C_{2} \rightarrow C=C_{1} \cup C_{2}
$$

be the partial normalization map at $u$, and put $\left\{u_{1}, u_{2}\right\}:=\mathfrak{n}^{-1}(u)$ with $u_{i} \in C_{i}$.

We assign a Young diagram $\Delta^{(i)}$ of type $\leq(r, s)$ to each $p^{(i)}$ for $1 \leq i \leq m$, and $\Gamma^{(j)}$ to each $q^{(j)}$ for $1 \leq j \leq n$. Assume that

$$
\sum_{i=1}^{m}\left|\Delta^{(i)}\right| \equiv \sum_{j=1}^{n}\left|\Gamma^{(j)}\right| \quad(\bmod 2),
$$

and put $\eta=(-1)^{\sum_{i=1}^{m}\left|\Delta^{(i)}\right|}$. Put

$$
\begin{aligned}
& \mathcal{H}^{\widehat{\mathfrak{s p}_{2 r}}}(\vec{\Delta} ; s):=\bigotimes_{i=1}^{m} \widehat{\mathcal{H}^{\mathfrak{\mathfrak { s p } _ { 2 r }}}}\left(\Delta^{(i)} ; s\right), \quad \mathcal{H}^{\widehat{\mathfrak{s p}_{2 r}}}(\vec{\Gamma} ; s):=\bigotimes_{j=1}^{n} \widehat{\mathcal{H}^{\left(\mathfrak{s p}_{2 r}\right.}}\left(\Gamma^{(j)} ; s\right), \\
& \mathcal{H}^{\widehat{\mathfrak{s p}_{2 s}}}\left(\vec{\Delta}^{*} ; r\right):=\bigotimes_{i=1}^{m} \widehat{\mathcal{H}^{\widehat{\mathfrak{p}_{2 s}}}}\left(\Delta^{(i) *} ; s\right), \quad \mathcal{H}^{\widehat{\mathfrak{s p}_{2 s}}}\left(\vec{\Gamma}^{*} ; s\right):=\bigotimes_{j=1}^{n} \widehat{\mathcal{H}^{\widehat{\mathfrak{s p}_{2 s}}}}\left(\Gamma^{(j) *} ; s\right) \\
& \mathcal{H}^{\widehat{\mathfrak{s o} 2 N}}\left(\overrightarrow{\epsilon_{1}} ; 1\right):=\bigotimes_{i=1}^{m} \widehat{\mathcal{H}^{\widehat{\mathfrak{s o}} 2 N}}\left((-1)^{\mid \Delta^{(i)}} ; 1\right), \quad \mathcal{H}^{\widehat{\mathfrak{s o} 2 N}}\left(\overrightarrow{\epsilon_{2}} ; 1\right):=\bigotimes_{j=1}^{n} \widehat{\mathcal{H}^{\widehat{\mathfrak{s o}} 2 N}}\left((-1)^{\left|\Gamma^{(j)}\right|} ; 1\right)
\end{aligned}
$$

Let

$$
\begin{aligned}
& \varphi^{(i)}: \widehat{\mathcal{H}^{\widehat{\mathfrak{p p}_{2 r}}}}\left(\Delta^{(i)} ; s\right) \otimes \widehat{\mathcal{H}^{\widehat{\mathfrak{s p}_{2 s}}}}\left(\Delta^{(i) *} ; r\right) \rightarrow \widehat{\mathcal{H}^{\widehat{\mathfrak{s o}_{2 N}}}}\left((-1)^{\left|\Delta^{(i)}\right|} ; 1\right),
\end{aligned}
$$

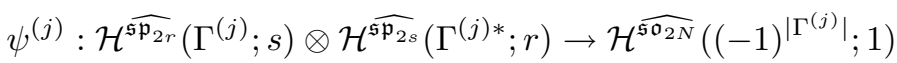

be non-zero morphisms of $\left(\widehat{\mathfrak{s p}_{2 r}} \oplus \widehat{\mathfrak{s p}_{2 s}}\right)$-modules.

If $A$ is a Young diagram of type $\leq(r, s)$, we have an isomorphism $V_{A} \simeq\left(V_{A}\right)^{\vee}$ of $\mathfrak{s p}_{2 r}$-modules. Hence there is a unique (up to scalar) nonzero morphism

$$
\mathbb{C} \rightarrow V_{A} \otimes V_{A}
$$

of $\mathfrak{s p}_{2 r}$-modules. Similarly we have morphisms

$$
\begin{aligned}
& \mathbb{C} \rightarrow V_{A^{*}} \otimes V_{A^{*}} \\
& \mathbb{C} \rightarrow V_{\eta} \otimes V_{\eta}
\end{aligned}
$$

of $\mathfrak{s p}_{2 s}$-modules and $\mathfrak{s o}_{2 N}$-modules respectively. (Here recall our convention that for $\mathfrak{s o}_{2 N}$-modules $V_{+1}=V_{\alpha}$ and $V_{-1}=V_{\beta}$.) 
Now consider the diagram

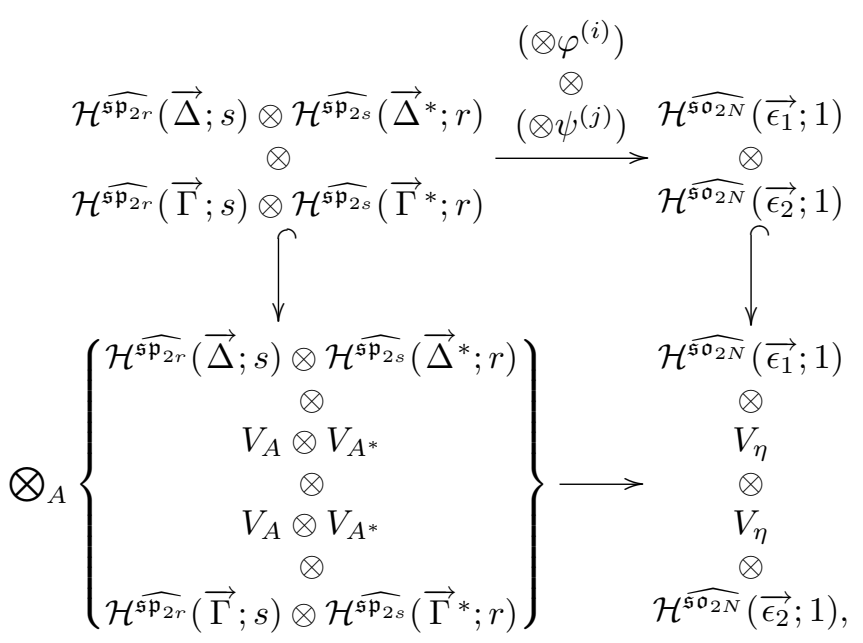

where $A$ runs through all Young diagrams of type $\leq(r, s)$ with $(-1)^{|A|}=\eta$, and the vertical arrows are induced by (3.13), (3.14) and (3.15), and the lower horizontal arrow is induced by (3.7) or (3.8).

Lemma 3.7. The above diagram is commutative, if necessary, after adjusting the non-zero morphism $V_{A} \otimes V_{A^{*}} \rightarrow V_{\eta}$ of $\left(\mathfrak{s p}_{2 r} \oplus \mathfrak{s p}_{2 s}\right)$-modules by scalar.

Proof. The morphism (3.15) induces an isomorphism $V_{\eta} \rightarrow V_{\eta}^{\vee}$ of $\mathfrak{s o}_{2 N}$-modules.

Composing it with the isomorphisms (3.7) or (3.8), we have an isomorphism

$$
\bigoplus_{A \text { s.t. }(-1)^{|A|}=\eta} V_{A} \otimes V_{A^{*}} \simeq \bigoplus_{A \text { s.t. }(-1)^{|A|}=\eta}\left(V_{A} \otimes V_{A^{*}}\right)^{\vee}
$$

of $\left(\mathfrak{s p}_{2 r} \oplus \mathfrak{s p _ { 2 s }}\right)$-modules. This is a direct sum of isomorphisms

$$
V_{A} \otimes V_{A^{*}} \rightarrow\left(V_{A} \otimes V_{A^{*}}\right)^{\vee}
$$

of $\left(\mathfrak{s p}_{2 r} \oplus \mathfrak{s p}_{2 s}\right)$-modules, which is induced by the tensor product of (3.13) and (3.14).

Put $\overrightarrow{p^{\prime}}:=\vec{p} \cup\left\{u_{1}\right\}, \overrightarrow{q^{\prime}}:=\vec{q} \cup\left\{u_{2}\right\}, \overrightarrow{\Delta^{\prime}}:=\vec{\Delta} \cup\{A\}, \overrightarrow{\Gamma^{\prime}}:=\vec{\Gamma} \cup\{A\}, \overrightarrow{\epsilon_{i}^{\prime}}:=\overrightarrow{\epsilon_{i}} \cup\{\eta\}$. The diagram (3.16) induces the following diagram:

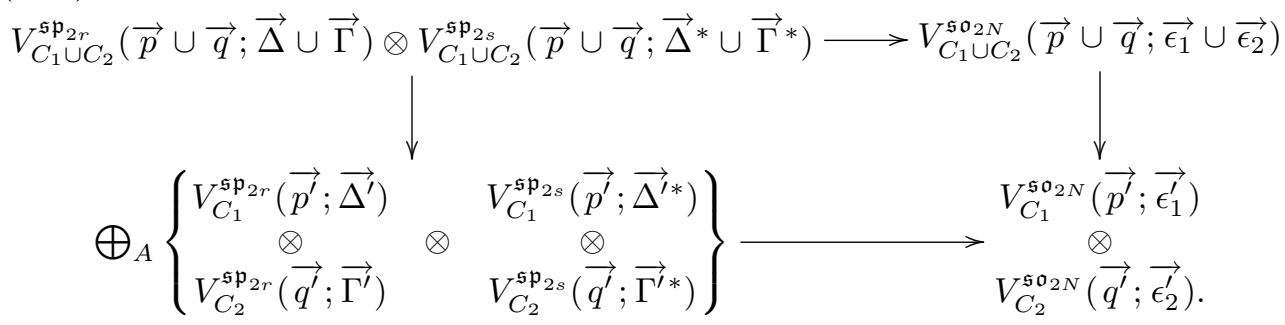

Assume that both $m$ and $n$ are odd. Then both the source and the target of the right vertical arrow are 1-dimensional, and it is an isomorphism. So we have the compatibility of the rank-level duality map and the factorization. 
Proposition 3.8. We have a commutative diagram:

(3.19)

$$
\begin{aligned}
& V_{C_{1} \cup C_{2}}^{\mathfrak{s p}_{2 r} \dagger}(\vec{p} \cup \vec{q} ; \vec{\Delta} \cup \vec{\Gamma})^{*} \longrightarrow V_{C_{1} \cup C_{2}}^{\mathfrak{s p}_{2 s} \dagger}\left(\vec{p} \cup \vec{q} ; \vec{\Delta}^{*} \cup \vec{\Gamma}^{*}\right)
\end{aligned}
$$

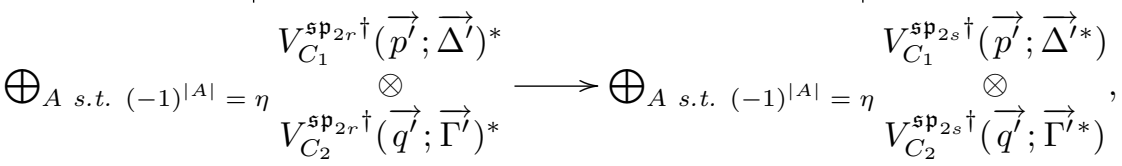

where the vertical arrows are factorization isomorphisms, and the horizontal arrows are rank-level duality maps.

\section{STRANGE DUALITY IS RANK-LEVEL DUALITY}

In this section we first recall the isomorphism of a space of generalized thetas and a conformal block. Then we shall show that by the isomorphism the strange duality for parabolic symplectic bundles is the rank-level duality for $\left(\mathfrak{s p}_{2 r}, \mathfrak{s p}_{2 s}\right)$.

4.1. Isomorphism of spaces of generalized thetas and conformal blocks. In this section we recall the isomorphism of a space of generalized thetas and a conformal block. Here we give a rather sketchy explanation. For details, see [L-S] (especially $\S 8.9$ ).

We use the notation listed at the beginning of Section 3.

Let $G$ be the simple and simply connected algebraic group with $T_{e} G=\mathfrak{g}$. Let $T$ be the maximal torus with $T_{e}(T)=\mathfrak{h}$, and $B$ the Borel subgroup such that $T_{e} B=\mathfrak{h} \oplus \bigoplus_{\alpha>0} \mathfrak{g}_{\alpha}$.

Let $C$ be a smooth projective curve, and $p^{(1)}, \ldots, p^{(m)}$ points of $C$. Let $P^{(1)}, \ldots, P^{(m)}$

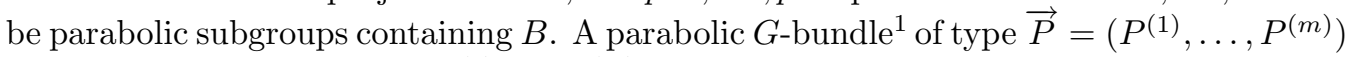
on a pointed curve $\left(C ; \vec{p}=\left(p^{(1)}, \ldots, p^{(m)}\right)\right)$ is a $G$-bundle $E$ on $C$ together with an element $\left.\sigma^{(j)} \in E\left(G / P^{(j)}\right)\right|_{p^{(j)}}$ for $1 \leq j \leq m$. Denote by $\mathcal{M}_{G}^{\text {Par }}(\vec{p} ; \vec{P})$ the moduli stack of parabolic $G$-bundles of type $\vec{P}$ on $(C ; \vec{p})$.

Fix a point $p \in C \backslash\left\{p^{(1)}, \ldots, p^{(m)}\right\}$ and an isomorphism $\widehat{\mathcal{O}_{C, p}} \simeq \mathbb{C}[[z]]$. Put $U:=C \backslash\{p\}, D:=\operatorname{Spec} \widehat{\mathcal{O}_{C, p}}$ and $D^{*}:=\operatorname{Spec} \operatorname{Frac}\left(\widehat{\mathcal{O}_{C, p}}\right)$.

Let $L G$ be the loop group of $G$, which is by definition the $\mathbb{C}$-group defined by $R \mapsto G(R((z)))$. The subgroup $L^{+} G$ of $L G$ is defined by $R \mapsto G(R[[z]])$. We denote by $\mathcal{Q}_{G}$ the quotient $\mathbb{C}$-space $L G / L^{+} G$. Put

$$
\mathcal{Q}_{G}^{P a r}(\vec{P}):=\mathcal{Q}_{G} \times \prod_{j=1}^{m} G / P^{(j)} .
$$

Given $\Phi \in G(\mathbb{C}((z)))$ and $a^{(j)} \in G / P^{(j)}$ for $1 \leq j \leq m$, if we glue two trivial $G$ bundles $G \times D$ and $G \times U$ on $D^{*}$ by $\Phi: G \times\left. D\right|_{D^{*}} \rightarrow G \times\left. U\right|_{D^{*}}$, we obtain a parabolic $G$-bundle of type $\vec{P}$ on $(C ; \vec{p})$. If we substitute $\Phi B$ for $\Phi$ with $B \in G(\mathbb{C}[[z]])$, then we obtain an isomorphic parabolic $G$-bundle. So we have a morphism

$$
\pi: \mathcal{Q}_{G}^{P a r}(\vec{P}) \rightarrow \mathcal{M}_{G}^{P a r}(\vec{p} ; \vec{P}) .
$$

The $\mathbb{C}$-group $L_{C} G$ defined by $R \mapsto G(R \otimes \mathcal{O}(U))$ acts on $\mathcal{Q}_{G}^{P a r}(\vec{P})$ from the left, and we have $L_{C} G \backslash \mathcal{Q}_{G}^{P a r}(\vec{P})=\mathcal{M}_{G}^{P a r}(\vec{p} ; \vec{P})([$ L-S, Theorem 8.5]).

\footnotetext{
${ }^{1}$ This is call a quasi-parabolic $G$-bundle in $[\mathrm{L}-\mathrm{S}]$
} 
By [L-S, Proposition 8.7], we have

$$
\operatorname{Pic} \mathcal{Q}_{G}^{P a r}(\vec{P})=\mathbb{Z} \mathcal{O}_{\mathcal{Q}_{G}}(1) \times \prod_{j=1}^{m} \operatorname{Pic}\left(G / P^{(j)}\right)
$$

By [L-S, Theorem 1.1], the map

$$
\pi^{*}: \operatorname{Pic} \mathcal{M}_{G}^{\text {Par }}(\vec{p} ; \vec{P}) \rightarrow \operatorname{Pic} \mathcal{Q}_{G}^{\text {Par }}(\vec{P})
$$

is bijective if $G$ is of classical or $G$ type.

As explained in [L-S, §4], there exists an extension of $L G$ by $\mathbb{G}_{\mathrm{m}}$

$$
0 \rightarrow \mathbb{G}_{\mathrm{m}} \rightarrow \widehat{L G} \rightarrow L G \rightarrow 0
$$

such that $\operatorname{Lie}(L G)=\widehat{\mathfrak{g}}$. We can express that $\mathcal{Q}_{G}=\widehat{L G} / \widehat{L^{+} G}$. The line bundle $\mathcal{O}_{\mathcal{Q}_{G}}(1)$ has a $\widehat{L G}$-linearization. So the vector space $\mathrm{H}^{0}\left(\mathcal{Q}_{G}, \mathcal{O}_{\mathcal{Q}_{G}}(l)\right)$ becomes a $\widehat{\mathfrak{g}}$-module. There is an isomorphism

$$
\mathrm{H}^{0}\left(\mathcal{Q}_{G}, \mathcal{O}_{\mathcal{Q}_{G}}(l)\right) \simeq \mathcal{H}^{\widehat{\mathfrak{g}}}(0 ; l)^{*}
$$

of $\widehat{\mathfrak{g}}$-modules. (See [L-S, §4] for details.)

Fix a positive integer $l$, and $\vec{\lambda}:=\left(\lambda^{(1)}, \ldots, \lambda^{(m)}\right) \in P_{l}^{\oplus m}$. Assume that each $\lambda^{(j)}$, considered as a character of $T$, extends to a character of the parabolic subgroup $P^{(j)}$. We denote by $\mathcal{L}_{-\lambda^{(j)}}$ the line bundle on $G / P^{(j)}$ associated to the character $-\lambda^{(j)}: P^{(j)} \rightarrow \mathbb{C}^{\times}$. We denote by $\mathcal{L}(l ; \vec{\lambda})$ the line bundle on $\mathcal{M}_{G}^{\text {Par }}(\vec{p} ; \vec{P})$ such that $\pi^{*} \mathcal{L}(l ; \vec{\lambda})=\mathcal{O}_{\mathcal{Q}_{G}}(l) \otimes \bigotimes_{j=1}^{m} \mathcal{L}_{-\lambda^{(j)}}$. In this paper, we are concerned with the case of $G$ being of type $C$ or $D$. If $G$ is of type $C$, then $\mathcal{L}(1 ; \overrightarrow{0})$ is the determinant line bundle. If $G$ is of type $D$, then $\mathcal{L}(1 ; \overrightarrow{0})$ is the pfaffian line bundle (cf [L-S, Theorem1.1]).

The line bundle $\pi^{*} \mathcal{L}(l ; \vec{\lambda})$ on $\mathcal{Q}_{G}^{\text {Par }}(\vec{P})$ has a $L_{C} G$-linearization. The vector space of global sections

$$
\mathrm{H}^{0}\left(\mathcal{Q}_{G}^{P a r}(\vec{P}), \pi^{*} \mathcal{L}(l ; \vec{\lambda})\right)=\mathrm{H}^{0}\left(\mathcal{Q}_{G}, \mathcal{O}_{\mathcal{Q}_{G}}(l)\right) \otimes \bigotimes_{j=1}^{m} \mathrm{H}^{0}\left(G / P^{(j)}, \mathcal{L}_{-\lambda^{(j)}}\right)
$$

becomes a $\mathfrak{g} \otimes \mathcal{O}(U)$-module by differentiating the $L_{C} G$-action, which, by (4.1), turns out to be canonically isomorphic as a $\mathfrak{g} \otimes \mathcal{O}(U)$-module to

$$
\mathcal{H}^{\widehat{\mathfrak{g}}}(0 ; l)^{*} \otimes \bigotimes_{j=1}^{m} V_{\lambda^{(j)}}^{*}
$$

where the $\mathfrak{g} \otimes \mathcal{O}(U)$-module structure is given by

$$
\begin{aligned}
(X \otimes f)\left(\theta \otimes \varphi_{1} \otimes \cdots \otimes \varphi_{m}\right)= & \left(\left(X \otimes f_{p}\right) \theta\right) \otimes \varphi_{1} \otimes \cdots \otimes \varphi_{m} \\
& +\sum_{j=1}^{m} f\left(p^{(j)}\right) \varphi_{1} \otimes \cdots \otimes\left(X \varphi_{j}\right) \otimes \cdots \otimes \varphi_{m} .
\end{aligned}
$$


We have isomorphisms

$$
\begin{aligned}
& \mathrm{H}^{0}\left(\mathcal{M}_{G}^{P a r}(\vec{p} ; \vec{P}), \mathcal{L}(l ; \vec{\lambda})\right) \simeq \mathrm{H}^{0}\left(\mathcal{Q}_{G}^{P a r}(\vec{P}), \mathcal{O}(l) \otimes \bigotimes_{j=1}^{m} \mathcal{L}_{-\lambda^{(j)}}\right)^{L_{C} G} \\
& \simeq\left\{\mathrm{H}^{0}\left(\mathcal{Q}_{G}, \mathcal{O}(l)\right) \otimes \bigotimes_{j=1}^{m} \mathrm{H}^{0}\left(G / P^{(j)}, \mathcal{L}_{-\lambda^{(j)}}\right)\right\}^{L_{C} G} \\
& \simeq\left\{\mathrm{H}^{0}\left(\mathcal{Q}_{G}, \mathcal{O}(l)\right) \otimes \bigotimes_{j=1}^{m} \mathrm{H}^{0}\left(G / P^{(j)}, \mathcal{L}_{-\lambda^{(j)}}\right)\right\}^{\mathfrak{g} \otimes \mathcal{O}(U)} \\
& \simeq\left\{\mathcal{H}^{\widehat{\mathfrak{g}}}(0 ; l)^{*} \otimes \bigotimes_{j=1}^{m} V_{\lambda^{(j)}}^{*}\right\}^{\mathfrak{g} \otimes \mathcal{O}(U)}
\end{aligned}
$$

As explained in Section 3.1, we have isomorphisms

$$
\begin{aligned}
& \left\{\mathcal{H}^{\widehat{\mathfrak{g}}}(0 ; l)^{*} \otimes \bigotimes_{j=1}^{m} V_{\lambda^{(j)}}^{*}\right\}^{\mathfrak{g} \otimes \mathcal{O}(U)} \simeq\left\{\mathcal{H}^{\widehat{\mathfrak{g}}}(0 ; l)^{*} \otimes \bigotimes_{j=1}^{m} \mathcal{H}^{\widehat{\mathfrak{g}}}\left(\lambda^{(j)} ; l\right)^{*}\right\}^{\mathfrak{g} \otimes \mathcal{O}\left(U \backslash\left\{p^{(1)}, \ldots, p^{(m)}\right\}\right)} \\
& \stackrel{\simeq}{\longrightarrow}\left\{\bigotimes_{j=1}^{m} \mathcal{H}^{\widehat{\mathfrak{g}}}\left(\lambda^{(j)} ; l\right)^{*}\right\}^{\mathfrak{g} \otimes \mathcal{O}\left(C \backslash\left\{p^{(1)}, \ldots, p^{(m)}\right\}\right)} \\
& =V_{C}^{\mathfrak{g} \dagger}(\vec{p} ; \vec{\lambda}) \text {. }
\end{aligned}
$$

Combining the isomorphisms (4.3) and (4.4), we have an isomorphism

$$
\mathrm{H}^{0}\left(\mathcal{M}_{G}^{P a r}(\vec{p} ; \vec{P}), \mathcal{L}(l ; \vec{\lambda})\right) \simeq V_{C}^{\mathfrak{g} \dagger}(\vec{p} ; \vec{\lambda}) .
$$

4.2. Reformulation of the strange duality for parabolic symplectic bundles. In this section we reformulate the strange duality in terms of Sp-bundles, not vector bundles with a non-degenerate alternate form. We use the notation in Section 3.4. Moreover we assume that $\mathbf{f}_{N}=\mathbf{e}_{r+1} \otimes \mathbf{g}_{s}$.

We denote by $\left(W_{2 r}\right)_{\mathrm{std} \bullet},\left(W_{2 s}\right)_{\mathrm{std} \bullet}$ and $\left(W_{2 N}\right)_{\mathrm{std} \bullet}$ respectively the standard filtrations

$$
\begin{aligned}
& <\mathbf{e}_{1}, \ldots, \mathbf{e}_{r}>\supset<\mathbf{e}_{1}, \ldots, \mathbf{e}_{r-1}>\cdots \supset<\mathbf{e}_{1}, \mathbf{e}_{2}>\supset<\mathbf{e}_{1}>\supset 0 \\
& <\mathbf{g}_{1}, \ldots, \mathbf{g}_{s}>\supset<\mathbf{g}_{1}, \ldots, \mathbf{g}_{s-1}>\cdots \supset<\mathbf{g}_{1}, \mathbf{g}_{2}>\supset<\mathbf{g}_{1}>\supset 0, \\
& <\mathbf{f}_{1}, \ldots, \mathbf{f}_{N}>\supset<\mathbf{f}_{1}, \ldots, \mathbf{f}_{N-1}>\cdots \supset<\mathbf{f}_{1}, \mathbf{f}_{2}>\supset<\mathbf{f}_{1}>\supset 0 .
\end{aligned}
$$

Let $B_{2 r}$ and $B_{2 s}$ be the subgroups of $\operatorname{Sp}\left(W_{2 r}\right)$ and $\operatorname{Sp}\left(W_{2 s}\right)$ that stabilize the filtrations $\left(W_{2 r}\right)_{\text {std }}$ and $\left(W_{2 s}\right)_{\text {std }}$ respectively. Let $B \subset \mathrm{SO}\left(W_{2 N}\right)$ be the subgroup that stabilizes the filtration $\left(W_{2 N}\right)_{\text {std }}$. For a Young diagram $\Lambda$ of type $\leq(r, s)$, we denote by $P_{\Lambda} \subset \mathrm{SO}\left(W_{2 N}\right)$ the stabilizer group of the maximal isotropic vector subspace $\mu_{\Lambda}\left(\left(W_{2 r}\right)_{\text {std } \bullet},\left(W_{2 s}\right)_{\text {std } \bullet}\right) \subset W_{2 N}$. By the assumption that $\mathbf{f}_{N}=\mathbf{e}_{r+1} \otimes \mathbf{g}_{s}$, both the parabolic subgroups $P_{(0, \ldots, 0)}$ and $P_{(1,0, \ldots, 0)}$ contain the Borel subgroup $B$. We write $P_{+1}$ and $P_{-1}$ instead of $P_{(0, \ldots, 0)}$ and $P_{(1,0, \ldots, 0)}$ respectively.

Let

$$
c: \operatorname{Spin}\left(W_{2 N}\right) \rightarrow \mathrm{SO}\left(W_{2 N}\right)
$$

be the canonical covering map. Let

$$
t: \operatorname{Sp}\left(W_{2 r}\right) \times \operatorname{Sp}\left(W_{2 s}\right) \rightarrow \mathrm{SO}\left(W_{2 N}\right)
$$


be the tensor morphism. Since the symplectic groups are simply connected, there is a unique morphism

$$
\tilde{t}: \operatorname{Sp}\left(W_{2 r}\right) \times \operatorname{Sp}\left(W_{2 s}\right) \rightarrow \operatorname{Spin}\left(W_{2 N}\right)
$$

such that $c \circ \tilde{t}=t$. In the following, for a subgroup $H \subset \mathrm{SO}\left(W_{2 N}\right), \widetilde{H}$ denotes $c^{-1}(H)$.

For a Young diagram $\Lambda$ of type $\leq(r, s)$, let

$$
\mu_{\Lambda}^{\prime}: \operatorname{Sp}\left(W_{2 r}\right) / B_{2 r} \times \operatorname{Sp}\left(W_{2 s}\right) / B_{2 s} \rightarrow \operatorname{Spin}\left(W_{2 N}\right) / \widetilde{P_{(-1)^{|\Lambda|}}}=\operatorname{SO}\left(W_{2 N}\right) / P_{(-1)^{|\Lambda|}}
$$

be the morphism determined by the commutative diagram

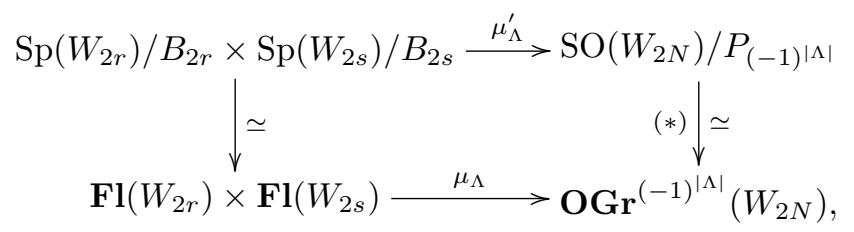

where $(*)$ is the SO-equivariant isomorphism such that $1 \mapsto \mu_{(0, \ldots, 0)}\left(\left(W_{2 r}\right)_{\operatorname{std} \bullet},\left(W_{2 s}\right)_{\text {std }}\right)$ if $|\Lambda|$ is even, and $1 \mapsto \mu_{(1,0, \ldots, 0)}\left(\left(W_{2 r}\right)_{\text {std } \bullet},\left(W_{2 s}\right)_{\text {std } \bullet}\right)$ if $|\Lambda|$ is odd.

Let $(C ; \vec{p})=\left(p^{(1)}, \ldots, p^{(m)}\right)$ be a smooth projective curve with smooth points. Each point $p^{(j)}$ is assigned a Young diagram $\Lambda^{(j)}$ of type $\leq(r, s)$.

Put $\vec{\epsilon}:=\left(\epsilon^{(1)}, \ldots, \epsilon^{(m)}\right)$ with $\epsilon^{(j)}=(-1)^{\left|\Lambda^{(j)}\right|}$, and $\overrightarrow{P_{\epsilon}}:=\left(P_{\epsilon^{(1)}}, \ldots, P_{\epsilon^{(m)}}\right)$, $\overrightarrow{B_{2 r}}:=\left(B_{2 r}, \ldots, B_{2 r}\right)$ and $\overrightarrow{B_{2 s}}:=\left(B_{2 s}, \ldots, B_{2 s}\right)$. Let

$$
\tau_{(C ; \vec{\Lambda})}^{\prime}: \mathcal{M}_{\mathrm{Sp}_{2 r}}^{\text {Par }}\left(\vec{p} ; \overrightarrow{B_{2 r}}\right) \times \mathcal{M}_{\mathrm{Sp}_{2 s}}^{\text {Par }}\left(\vec{p} ; \overrightarrow{B_{2 s}}\right) \rightarrow \mathcal{M}_{\mathrm{Spin}_{2 N}}^{\text {Par }}\left(\vec{p} ; \overrightarrow{P_{\epsilon}}\right)
$$

be the morphism of stacks that associates to a pair

$$
\left(E,\left\{\left.a_{j} \in E\left(\mathrm{Sp}_{2 r} / B_{2 r}\right)\right|_{p^{(j)}}\right\}_{1 \leq j \leq m} ; F,\left\{\left.b_{j} \in F\left(\mathrm{Sp}_{2 s} / B_{2 s}\right)\right|_{p^{(j)}}\right\}_{1 \leq j \leq m}\right)
$$

the object

$$
\left(\left(E \times_{C} F\right)\left(\operatorname{Spin}_{2 \mathrm{~N}}\right),\left\{\mu_{\Lambda^{(j)}}^{\prime}\left(\left(a_{j}, b_{j}\right)\right) \in\left(E \times_{C} F\right)\left(\operatorname{Spin}_{2 N} / \widetilde{\left.P_{\epsilon^{(j)}}\right)}\right\}_{1 \leq j \leq m}\right) .\right.
$$

Assuming that $m$ is even, let us compare $\tau_{(C ; \vec{\Lambda})}^{\prime}$ in the above and $\tau_{(C ; \vec{\Lambda})}$ in $(2.2)$. Given an object

$$
\left(E,\left\{\left.a_{j} \in E\left(\mathrm{Sp}_{2 r} / B_{2 r}\right)\right|_{p^{(j)}}\right\}_{1 \leq j \leq m}\right) \in \mathcal{M}_{\mathrm{Sp}_{2 r}}^{P a r}\left(\vec{p} ; \vec{B}_{2 r}\right),
$$

by considering the standard representation of $\mathrm{Sp}_{2 r}$ and the isomorphism $\mathrm{Sp}_{2 r} / B_{2 r} \simeq$ $\mathbf{F l}\left(\mathbb{C}^{2 r}\right)$ given by $1 \mapsto\left(W_{2 r}\right)_{\text {std } \bullet}$, we obtain a parabolic symplectic bundle (in the sense of Definition 2.4). Conversely, from a parabolic symplectic bundle, we can construct a parabolic $\mathrm{Sp}_{2 r}$-bundle of type $\overrightarrow{B_{2 r}}$. Thus we have as isomorphism

$$
\mathcal{M}_{\mathrm{Sp}_{2 r}}^{\mathrm{Par}}\left(\vec{p} ; \overrightarrow{B_{2 r}}\right) \simeq M_{2 r}\left(C, \vec{p} ; \mathcal{O}_{C}\right)
$$

By the isomorphism, the line bundle $\mathcal{L}(s ; \vec{\Lambda})$ on $\mathcal{M}_{\mathrm{Sp}_{2 r}}^{\text {Par }}\left(\vec{p} ; \overrightarrow{B_{2 r}}\right)$ is isomorphic to the line bundle $\Xi^{(s ; \vec{\Lambda})}$ on $M_{2 r}\left(C, \vec{p} ; \mathcal{O}_{C}\right)$.

Since we are assuming that $m$ is even, we can take a square root of the line bundle $\omega_{C}(\vec{p})$. We fix a square root $\left(\omega_{C}(\vec{p})\right)^{\otimes \frac{1}{2}}$ and an isomorphism $\left(\left(\omega_{C}(\vec{p})\right)^{\otimes \frac{1}{2}}\right)^{\otimes 2} \simeq$ $\omega_{C}(\vec{p})$.

Then the above construction plus tensoring by $\left(\omega_{C}(\vec{p})\right)^{\otimes \frac{1}{2}}$ gives rise to an isomorphism of stacks

$$
\mathcal{M}_{\mathrm{Sp}_{2 s}}^{P a r}\left(\vec{p} ; \overrightarrow{B_{2 s}}\right) \simeq M_{2 s}\left(C, \vec{p} ; \omega_{C}(\vec{p})\right)
$$


Given an object

$$
\left(F,\left\{\left.c_{j} \in F\left(\operatorname{Spin}_{2 N} / \widetilde{P}_{\epsilon^{(j)}}\right)\right|_{p^{(j)}}\right\}_{1 \leq j \leq m}\right) \in \mathcal{M}_{\operatorname{Spin}_{2 N}}^{P_{1}}\left(\vec{p} ; \overrightarrow{P_{\epsilon}}\right),
$$

by considering the standard representation of $\mathrm{SO}$, and the isomorphism $(*)$, we obtain an orthogonal bundle $F\left(W_{2 N}\right)$ with values in $\mathcal{O}_{C}$, together with, for each $1 \leq j \leq m$, a maximal isotropic subspace $\left.U^{(j)} \subset F\left(W_{2 N}\right)\right|_{p^{(j)}}$. By associating to the object (4.10) the orthogonal bundle with value in $\omega_{C}$

$$
\operatorname{Ker}\left(\left.F\left(W_{2 N}\right) \rightarrow \bigoplus_{j=1}^{m} F\left(W_{2 N}\right)\right|_{p^{(j)}} / U^{(j)}\right) \otimes\left(\omega_{C}(\vec{p})\right)^{\otimes \frac{1}{2}},
$$

we obtain a morphism of stacks

$$
\xi: \mathcal{M}_{\text {Spin }}^{\text {Par }}\left(\vec{p} ; \overrightarrow{P_{\epsilon}}\right) \rightarrow N_{2 N}\left(C ; \omega_{C}\right) .
$$

By construction, the diagram

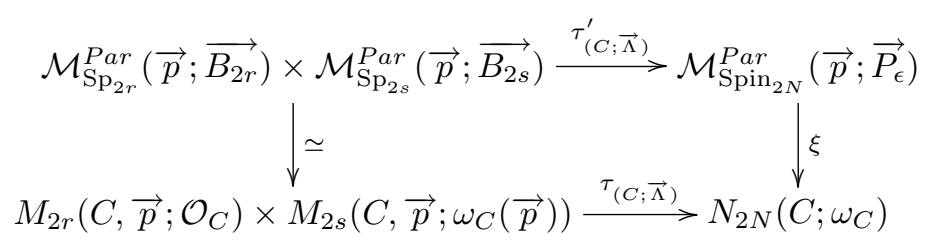

is commutative, and $\xi^{*} \mathcal{P} \simeq \mathcal{L}(1 ; \vec{\epsilon})$, where $\mathcal{P}$ is the pfaffian bundle, and we understand $+1=\alpha$ and $-1=\beta$ as before.

By Lemma 2.7, we have

$$
\operatorname{Im} \xi \subset N_{2 N}^{\prod_{j=1}^{m} \epsilon^{(j)}}\left(C ; \omega_{C}\right)
$$

Now we assume moreover that $C=\mathbb{P}^{1}$ and $\prod_{j=1}^{m} \epsilon^{(j)}=1$. Then by Corollary 3.4, the dimension of the conformal block $V_{\mathbb{P}^{\mathfrak{s o}_{2 N}}}^{\dagger}(\vec{p} ; \vec{\epsilon})$ of level one is one. So we have

$$
\operatorname{dim} \mathrm{H}^{0}\left(\mathcal{M}_{\operatorname{Spin}_{2 N}}^{P a r}\left(\vec{p} ; \overrightarrow{P_{\epsilon}}\right), \mathcal{L}(1 ; \vec{\epsilon})\right)=1 .
$$

Abbreviating $\mathcal{M}_{\mathrm{Sp}_{2 r}}^{\text {Par }}\left(\vec{p} ; \overrightarrow{B_{2 r}}\right), \mathcal{M}_{\mathrm{Sp}_{2 s}}^{\text {Par }}\left(\vec{p} ; \overrightarrow{B_{2 s}}\right), \mathcal{M}_{\mathrm{Spin}_{2 N}}^{\text {Par }}\left(\vec{p} ; \overrightarrow{P_{\epsilon}}\right), M_{2 r}\left(C, \vec{p} ; \mathcal{O}_{C}\right)$ and $M_{2 s}\left(C, \vec{p} ; \omega_{C}(\vec{p})\right)$ to $\mathcal{M}_{\mathrm{Sp}_{2 r}}^{P a r}, \mathcal{M}_{\mathrm{Sp}_{2 s}}^{P a r}, \mathcal{M}_{\mathrm{Spin}_{2 N}}^{P a r}, M_{2 r}$ and $M_{2 s}$ respectively, we have a commutative diagram

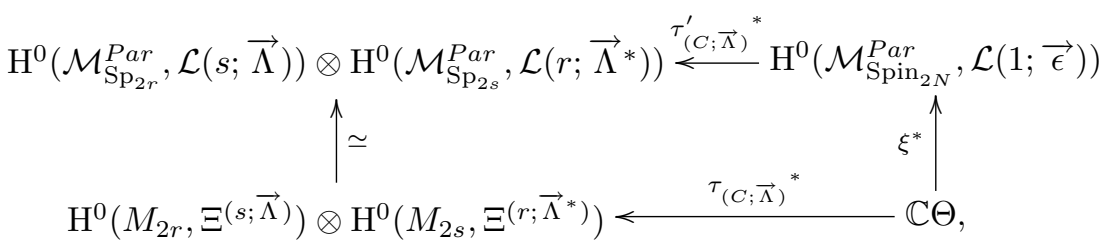

where $\Theta$ is the canonical section of the pfaffian bundle.

Lemma 4.1. The pull-back $\xi^{*} \Theta$ is not zero.

Proof. We find a parabolic $\operatorname{Spin}_{2 N^{-}}$bundles

$$
\mathbb{F}=\left(F,\left.\sigma_{j} \in F\left(\operatorname{Spin}_{2 N} / \widetilde{P_{\epsilon^{(j)}}}\right)\right|_{p^{(j)}}(1 \leq j \leq m)\right)
$$

such that the pfaffian section $\Theta$ does not vanish at $\xi(\mathbb{F})$. We assume that $\epsilon^{(j)}=1$ for $1 \leq j \leq 2 a$, and $\epsilon^{(j)}=-1$ for $2 a<j \leq m$. 
Consider the $\mathrm{SO}_{2 N}$-bundle $\bar{F}:=W_{2 N} \otimes_{\mathbb{C}} \mathcal{O}_{\mathbb{P}^{1}}$. We define the maximal isotropic subspaces $U_{+}, U_{+}^{\prime}, U_{-}, U_{-}^{\prime}$ of $W_{2 N}$ by

$$
\begin{array}{ll}
U_{+}:=\left\{\mathbf{f}_{1}, \ldots, \mathbf{f}_{N}\right\} & U_{+}^{\prime}:=\left\{\mathbf{f}_{N+1}, \ldots, \mathbf{f}_{2 N}\right\} \\
U_{-}:=\left\{\mathbf{f}_{1}, \ldots, \mathbf{f}_{N-1}, \mathbf{f}_{2 N}\right\} & U_{-}^{\prime}:=\left\{\mathbf{f}_{N+1}, \ldots, \mathbf{f}_{2 N-1}, \mathbf{f}_{N}\right\} .
\end{array}
$$

To each point $p^{(j)}$ we assign a maximal isotropic subspace $U^{(j)}$ of $\left.\bar{F}\right|_{p^{(j)}}$ so that $U^{(j)}$ is $U_{+}$for $1 \leq j \leq a, U_{+}^{\prime}$ for $a<j \leq 2 a, U_{-}$for $2 a<j \leq m / 2+a$, and $U_{-}^{\prime}$ for $m / 2+a<j \leq m$. Then the vector bundle

$$
\operatorname{Ker}\left(\left.\bar{F} \rightarrow \bigotimes_{j=1}^{m} \bar{F}\right|_{p^{(j)}} / U^{(j)}\right) \otimes \omega_{\mathbb{P}^{1}}(\vec{p})
$$

is a direct sum of $\mathcal{O}_{\mathbb{P}^{1}}(-1)$, so its $\mathrm{H}^{0}$ is zero. This means that the pfaffian section $\Theta$ does not vanish there.

It remains to show that the $\mathrm{SO}_{2 N}$-bundle $\bar{F}$ comes from a $\mathrm{Spin}_{2 N}$-bundle, but this is clear because it is a trivial bundle.

By the lemma, $\xi^{*}$ is an isomorphism. So we have a commutative diagram

$$
\begin{gathered}
\mathrm{H}^{0}\left(\mathcal{M}_{\mathrm{Sp}_{2 r}}^{\text {Par }}, \mathcal{L}(s ; \vec{\Lambda})\right)^{\vee} \stackrel{(\sharp)}{\longrightarrow} \mathrm{H}^{0}\left(\mathcal{M}_{\mathrm{Sp}_{2 s}}^{\text {Par }}, \mathcal{L}\left(r ; \vec{\Lambda}^{*}\right)\right) \\
\downarrow \\
\mathrm{H}^{0}\left(M_{2 r}, \Xi^{(s ; \vec{\Lambda})}\right)^{\vee} \longrightarrow \mathrm{H}^{0}\left(M_{2 s}, \Xi^{\left(r ; \vec{\Lambda}^{*}\right)}\right),
\end{gathered}
$$

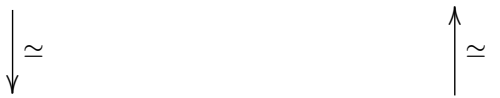

where $(\sharp)$ is induced by $\tau_{(C ; \vec{\Lambda})}^{\prime}{ }^{*}$, and the lower horizontal arrow is induced by $\tau_{(C ; \vec{\Lambda})}{ }^{*}$, that is, it is the map $(2.4)$.

The map $(\sharp)$ is a reformulation of the strange duality map for parabolic symplectic bundles in terms of Sp-bundles.

4.3. Strange duality is rank-level duality. In the previous section, when $m$ (the number of marked points) is even, we reformulated the strange duality for parabolic symplectic bundles on $\left(\mathbb{P}^{1} ; \vec{p}\right)$ in terms of parabolic Sp-bundles. In this section we show that the reformulated strange duality map $(\sharp)$ in $(4.13)$ is equal to the rank-level duality map (3.12).

Proposition 4.2. Let $\left(\mathbb{P}^{1} ; \vec{p}=\left(p^{(1)}, \ldots, p^{(m)}\right)\right.$ be a pointed $\mathbb{P}^{1}$. Let $\Lambda^{(j)}(1 \leq j \leq$ $m)$ be a Young diagram of type $\leq(r, s)$. Assume that both $m$ and $\sum_{j=1}^{m}\left|\Lambda^{(j)}\right|$ are even. Then the following diagram is commutative.

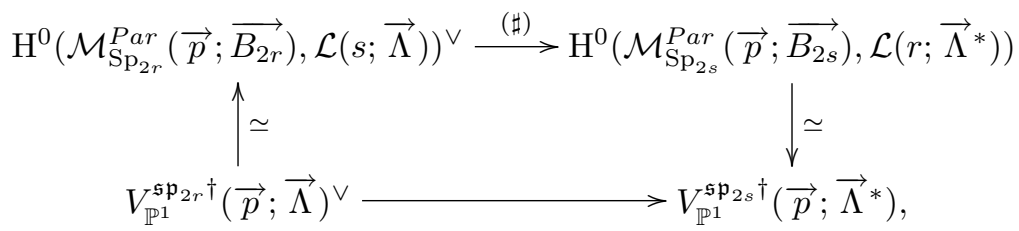

where the lower horizontal arrow is (3.12), and the vertical arrows are (4.5).

By this proposition and the commutative diagram (4.13), we know that the strange duality map (2.4) is an isomorphism if and only if the rank-level duality map (3.12) is an isomorphism.

We denote by $\nu$ the morphism

induced by $\widetilde{t}: \mathrm{Sp}_{2 r} \times \mathrm{Sp}_{2 s} \rightarrow \operatorname{Spin}_{2 N}$.

$$
\nu: \mathcal{Q}_{\mathrm{Sp}_{2 r}} \times \mathcal{Q}_{\mathrm{Sp}_{2 s}} \rightarrow \mathcal{Q}_{\mathrm{Spin}_{2 N}}
$$


For the proof of the proposition, we need the following lemma:

Lemma 4.3. For a Young diagram $\Lambda$ of type $\leq(r, s)$, we have a commutative (up to scalar) diagram:

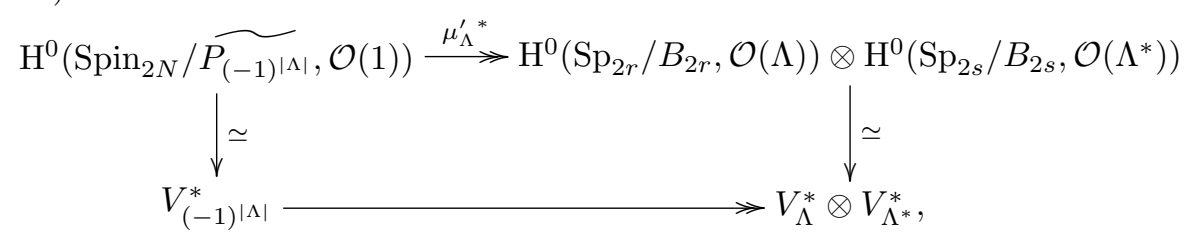

where the lower horizontal arrow is a nonzero $\left(\mathfrak{s p}_{2 r} \oplus \mathfrak{s p}_{2 s}\right)$-module homomorphism, which is unique up to scalar by (3.7) or (3.8).

Proof. The non-trivial part is the surjectivity of $\mu_{\Lambda}^{\prime *}$. This follows from $[\mathrm{A}$, Corollary 3.3.2].

Before starting the proof of Proposition 4.2, we observe the following:

By [Has, Theorem 2.4b (i) and Theorem 4.2], we have a $\left(\widehat{\mathfrak{s p}_{2 r}} \oplus \widehat{\mathfrak{s p}_{2 s}}\right)$-module homomorphism

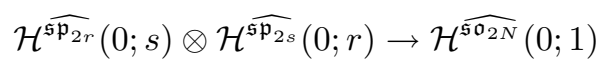

such that $v_{0} \otimes v_{0} \mapsto v_{0}$. So we have a commutative diagram

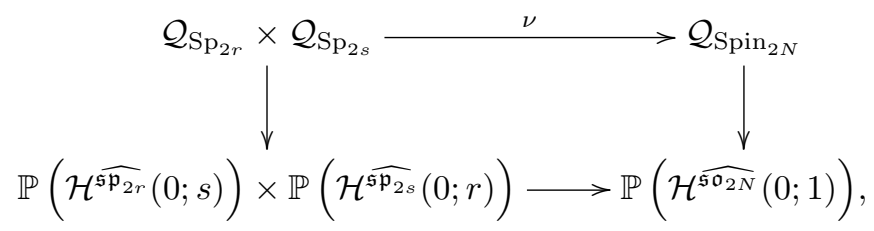

where the right vertical arrow is defined by

$$
\mathcal{Q}_{\operatorname{Spin}_{2 N}}=L \operatorname{Spin}_{2 N} / L^{+} \operatorname{Spin}_{2 N} \ni[g] \mapsto\left[g \cdot v_{0}\right] \in \mathbb{P}\left(\mathcal{H}^{\widehat{\mathfrak{s o}_{2 N}}}(0 ; 1)\right),
$$

and the left vertical arrow is defined similarly.

This induces a commutative diagram

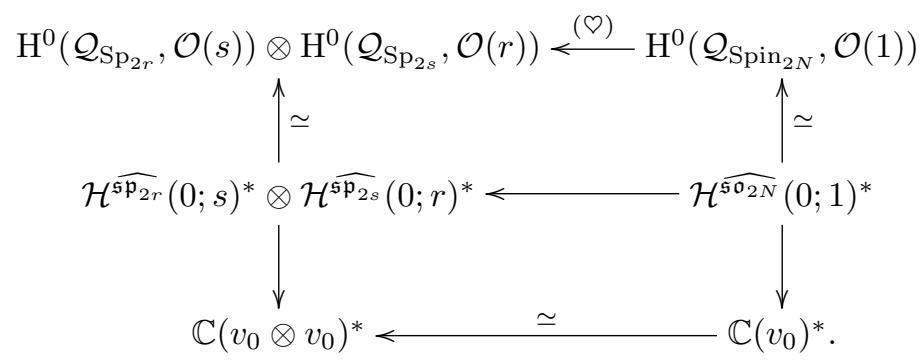

Now let us begin the proof of the proposition.

Proof of Proposition 4.2. The commutative diagram

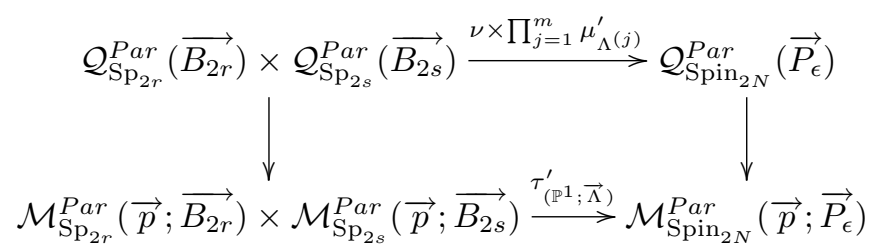


induces a commutative diagram

(4.19)

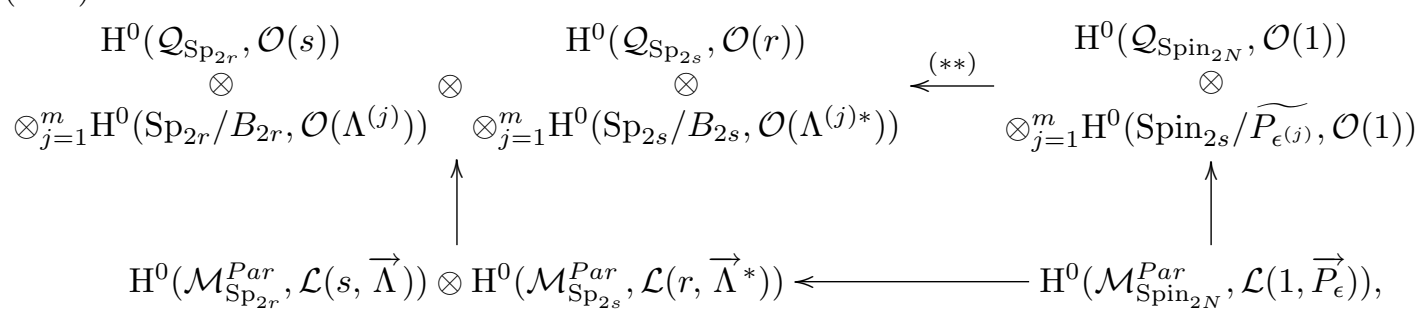

where $(* *)$ is a tensor product of $(\nabla)$ and $\mu_{\Lambda^{(j)}}^{\prime}{ }^{*}$. By the commutative diagrams (4.15) and (4.17), we have a commutative diagram

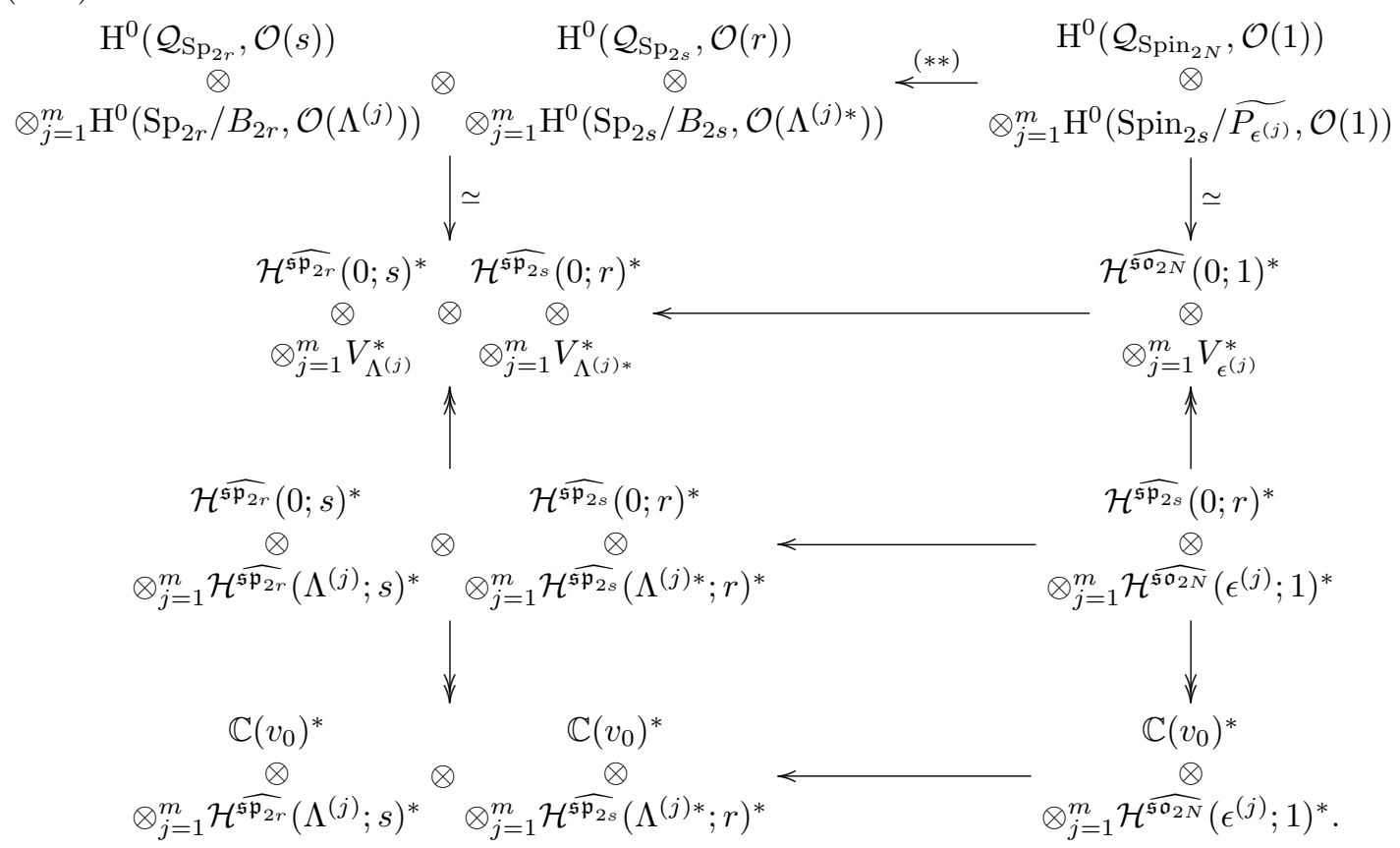

Combining the commutative diagrams (4.19) and (4.20), and tracing back the construction of the isomorphism (4.5), we obtain the commutativity of the diagram (4.14).

The following corollary claims that to see whether the rank-level duality map is an isomorphism or not, we may add even number of points and label them by the empty Young diagram:

Corollary 4.4. Let $\left(\mathbb{P}^{1} ; \vec{p}=\left(p^{(1)}, \ldots, p^{(m)}\right)\right)$ and $\vec{\Lambda}=\left(\Lambda^{(1)}, \ldots, \Lambda^{(m)}\right)$ be as in Proposition 4.2.

Let $p^{(m+1)}, \ldots, p^{\left(m^{\prime}\right)} \in \mathbb{P}^{1} \backslash \vec{p}$ be an even number of points, and $\Lambda^{(m+1)}, \ldots, \Lambda^{\left(m^{\prime}\right)}$ be the empty Young diagrams. Put

$$
\overrightarrow{p^{\prime}}=\left(p^{(1)}, \ldots, p^{\left(m^{\prime}\right)}\right) \quad \text { and } \quad \overrightarrow{\Lambda^{\prime}}=\left(\Lambda^{(1)}, \ldots, \Lambda^{\left(m^{\prime}\right)}\right) \text {. }
$$

Then the rank-level duality map

$$
V_{\mathbb{P}^{1}}^{\mathfrak{s p}_{2 r} \dagger}(\vec{p} ; \vec{\Lambda})^{\vee} \rightarrow V_{\mathbb{P}^{1}}^{\mathfrak{s p}_{2 s} \dagger}\left(\vec{p} ; \vec{\Lambda}^{*}\right)
$$

is an isomorphism if and only if

$$
V_{\mathbb{P}^{1}}^{\mathfrak{s p}_{2 r} \dagger}\left(\overrightarrow{p^{\prime}} ; \overrightarrow{\Lambda^{\prime}}\right)^{\vee} \rightarrow V_{\mathbb{P}^{1}}^{\mathfrak{s p}_{2 s} \dagger}\left(\overrightarrow{p^{\prime}} ; \overrightarrow{\Lambda^{\prime}}\right)
$$


is an isomorphism.

Proof. This is a consequence of Proposition 4.2 and Remark 2.10.

\section{KZ CONNECTION}

In this section we recall the $\mathrm{KZ}$ connection on a family of conformal blocks. We show that the rank-level duality map is compatible with the connections. This implies that if the rank-level duality map is an isomorphism for a pointed $\mathbb{P}^{1}$, then so is it for all pointed $\mathbb{P}^{1}$. In the non-parabolic case, this is proved in Belkale [Bel07].

We use the notation listed at the beginning of Section 3. Fix the level $l$.

5.1. KZ connection. For $X \in \mathfrak{g}$, we put $X(n):=X \otimes z^{n}$. The normal ordering $\circ \quad \circ$ is defined by

$$
\stackrel{\circ}{\circ}(n) Y(m) \stackrel{\circ}{\circ}= \begin{cases}X(n) Y(m) & \text { if } n<m \\ \frac{1}{2}(X(n) Y(m)+Y(m) X(n)) & \text { if } n=m \\ Y(m) X(n) & \text { if } n>m .\end{cases}
$$

We put

$$
L_{n}^{\widehat{\mathfrak{g}}}:=\frac{1}{2\left(g^{*}+l\right)} \sum_{k \in \mathbb{Z}} \sum_{a=1}^{\operatorname{dim} \mathfrak{g}} \circ J^{a}(k) J^{a}(n-k)_{\circ}^{\circ},
$$

where $\left\{J^{1}, J^{2}, \ldots\right\}$ is an orthonormal basis of $\mathfrak{g}$ with respect to the normalized Killing form.

Put

$$
B_{m}:=\left\{\left(z_{1}, \ldots, z_{m}\right) \in \mathbb{A}^{m} \mid z_{i} \neq z_{j}\right\} .
$$

Let $u$ be the affine coordinate of $\mathbb{A}^{1} \subset \mathbb{P}^{1}=\mathbb{A}^{1} \cup\{\infty\}$. Let

$$
\pi: \mathbb{P}^{1} \times B_{m} \rightarrow B_{m}
$$

be the projection, and $p^{(j)}$ be the section of $\pi$ given by

$$
B_{m} \ni\left(z_{1}, \ldots, z_{m}\right) \mapsto\left(z_{j},\left(z_{1}, \ldots, z_{m}\right)\right) \in \mathbb{P}^{1} \times B_{m} .
$$

Put $\xi_{j}:=u-z_{j}$, then $\xi_{j}$ vanishes along the section $p^{(j)}$ with order one. If $h$ is a meromorphic function on a neighborhood of the section $p^{(j)}$ having poles only along $p^{(j)}$, then $h$ can be expanded to a Laurent series with respect to $\xi$, which we denote by $t_{j}(h) \in \mathcal{O}_{B_{m}}\left(\left(\xi_{j}\right)\right)$.

Let $\vec{\Lambda}=\left(\Lambda^{(j)}, \ldots, \Lambda^{(m)}\right) \in P_{l}^{\oplus m}$. We can extend straightforwardly the definition of dual conformal blocks and conformal blocks for a fixed curve given in Section 3.1 to a family of curves, and we obtain a family of dual conformal blocks and a family of conformal blocks, which we denote by $V_{\mathbb{P}^{1} \times B_{m} / B_{m}}^{\mathfrak{g}}(\vec{p} ; \vec{\Lambda})$ and $V_{\mathbb{P}^{1} \times / B_{m}}^{\mathfrak{g} \dagger}(\vec{p} ; \vec{\Lambda})$ respectively. These are vector bundles on $B_{m}$.

The KZ connection on the vector bundle $V_{\mathbb{P}^{1} \times B_{m} / B_{m}}^{\mathfrak{g}}(\vec{p} ; \vec{\Lambda})$ is defined as follows. First we define a connection on $\mathcal{H}(\vec{\Lambda}) \otimes_{\mathcal{O}_{B_{m}}}=\mathcal{H}\left(\Lambda^{(1)}\right) \otimes_{\mathbb{C}} \cdots \otimes_{\mathbb{C}} \mathcal{H}\left(\Lambda^{(m)}\right) \otimes_{\mathbb{C}} \mathcal{O}_{B_{m}}$ by

$$
\begin{aligned}
\nabla_{\frac{\partial}{\partial z_{j}}}^{\widehat{\mathfrak{g}}}\left(\varphi_{1} \otimes \cdots \otimes \varphi_{m} \otimes f\right)= & \varphi_{1} \otimes \cdots \otimes \varphi_{m} \otimes \frac{\partial f}{\partial z_{j}} \\
& +\varphi_{1} \otimes \cdots \otimes L_{-1}^{\widehat{\mathfrak{g}}} \varphi_{j} \otimes \cdots \otimes \varphi_{m} \otimes f .
\end{aligned}
$$

Then by $\nabla_{\frac{\partial}{\partial z_{j}}}^{\widehat{\mathfrak{g}}}$, the subsheaf $\left(\mathfrak{g} \otimes \mathcal{O}\left(\mathbb{P}^{1} \times B_{m} \backslash \vec{p}\right)\right) \cdot \mathcal{H}(\vec{\Lambda}) \otimes \mathcal{O}_{B_{m}}$ is mapped into itself. So we have a connection

$$
\nabla^{\widehat{\mathfrak{g}}}: V_{\mathbb{P}^{1} \times B_{m} / B_{m}}^{\mathfrak{g}}(\vec{p} ; \vec{\Lambda}) \rightarrow V_{\mathbb{P}^{1} \times B_{m} / B_{m}}^{\mathfrak{g}}(\vec{p} ; \vec{\Lambda}) \otimes_{\mathcal{O}_{B_{m}}} \Omega_{B_{m}}^{1} .
$$

This is a flat connection and is called the $\mathrm{KZ}$ connection $([\mathrm{KZ}])$. 
The dual connection of $\nabla^{\widehat{\mathfrak{g}}}$ on $V_{\mathbb{P}^{1} \times B_{m} / B_{m}}^{\mathfrak{g} \dagger}(\vec{p} ; \vec{\Lambda})$ is also denoted by $\nabla^{\widehat{\mathfrak{g}}}$.

5.2. Compatibility of the connections for $\left(\mathfrak{s p}_{2 r} \oplus \mathfrak{s p}_{2 s}, \mathfrak{s o}_{4 r s}\right)$. We can construct the morphism (3.10) for the family $\pi: \mathbb{P}^{1} \times B_{m} \rightarrow B_{m}$ of $m$-pointed projective lines, and obtain

$$
V_{\mathbb{P}^{1} \times B_{m} / B_{m}}^{\mathfrak{s p _ { 2 r }}}(\vec{p} ; \vec{\Lambda}) \otimes_{\mathcal{O}_{B_{m}}} V_{\mathbb{P}^{1} \times B_{m} / B_{m}}^{\mathfrak{s p}_{2 s}}\left(\vec{p} ; \vec{\Lambda}^{*}\right) \rightarrow V_{\mathbb{P}^{1} \times B_{m} / B_{m}}^{\mathfrak{s o}_{2 N}}(\vec{p} ; \vec{\epsilon}) .
$$

The source has the flat connection $\nabla^{\widehat{\mathfrak{s p}_{2 r}}} \otimes \mathrm{id}+\mathrm{id} \otimes \nabla^{\widehat{\mathfrak{s p}_{2 s}}}$, and the target has $\nabla^{\widehat{\mathfrak{s o}_{2 N}}}$.

Proposition 5.1. The morphism (5.1) is compatible with the connection.

Proof. Let

$$
\widehat{\mathcal{H}^{\widehat{\mathfrak{p}_{2 r}}}}(\Lambda ; s) \otimes \mathcal{H}^{\widehat{\mathfrak{s p}_{2 s}}}\left(\Lambda^{*} ; r\right) \hookrightarrow \widehat{\mathcal{H}^{\widehat{\mathfrak{s o}_{2 N}}}}\left((-1)^{|\Lambda|} ; 1\right)
$$

be a non-zero $\left(\widehat{\mathfrak{s p}_{2 r}} \oplus \widehat{\mathfrak{s p}_{2 s}}\right)$-module homomorphism. By [Has, Proposition 4.1 (i)], for $\forall \varphi \otimes \theta \in \widehat{\mathcal{H}^{\widehat{\mathfrak{s p}_{2 r}}}}(\Lambda ; s) \otimes \mathcal{H}^{\widehat{\mathfrak{s p}_{2 s}}}\left(\Lambda^{*} ; r\right)$, we have

$$
L_{-1}^{\widehat{\mathfrak{s p}_{2 r}}}(\varphi) \otimes \theta+\varphi \otimes L_{-1}^{\widehat{\mathfrak{s p}_{2 s}}}(\theta)=\widehat{L_{-1}^{\mathfrak{s o n}_{2 N}}}(\varphi \otimes \theta) \text {. }
$$

Now the proposition follows from this equality and the definition of the KZ connection.

Corollary 5.2. If the rank-level duality map (3.12) is an isomorphism for an $m$ pointed $\mathbb{P}^{1}$, then it is an isomorphism for any $m$-pointed $\mathbb{P}^{1}$.

Proof. Let

$$
V_{\mathbb{P}^{1} \times B_{m} / B_{m}}^{\mathfrak{s p}_{2 r}^{\dagger}}(\vec{p} ; \vec{\Lambda})^{\vee} \otimes_{\mathcal{O}_{B_{m}}} V_{\mathbb{P}^{1} \times B_{m} / B_{m}}^{\mathfrak{s}_{2 N} \dagger}(\vec{p} ; \vec{\epsilon}) \rightarrow V_{\mathbb{P}^{1} \times B_{m} / B_{m}}^{\mathfrak{s p}_{2 s}^{\dagger}}\left(\vec{p} ; \vec{\Lambda}^{*}\right)
$$

be the family of the rank-level duality map. Both the source and the target have flat connections, and the map is compatible with the connections. If we locally take flat frames of the vector bundles, the map (5.2) is expressed by a constant matrix. Hence the rank of the map is constant over $B_{m}$.

\section{Proof of The RANK-LEVEL DUAlity FOR $\left(\mathfrak{s p}_{2 r}, \mathfrak{s p}_{2 s}\right)$}

In this section we prove the following theorem. The proof is similar to that of the rank-level duality for $\left(\widehat{\mathfrak{s l}}_{r}, \widehat{\mathfrak{s l}}_{l}\right)$ in $[\mathrm{NT}]$.

Theorem 6.1. Let $\left(\mathbb{P}^{1}, \vec{p}=\left(p^{(1)}, \ldots, p^{(m)}\right)\right)$ be an m-pointed $\mathbb{P}^{1}$, and $\vec{\Lambda}=$ $\left(\Lambda^{(1)}, \ldots, \Lambda^{(m)}\right)$ be an $m$-tuple of Young diagrams of type $\leq(r, s)$. Assume that both $m$ and $\sum_{j=1}^{m}\left|\Lambda^{(j)}\right|$ are even. Then the rank-level duality map

$$
V_{\mathbb{P}^{1}}^{\mathfrak{s p} \mathfrak{p}_{2 r}^{\dagger}}(\vec{p} ; \vec{\Lambda})^{\vee} \rightarrow V_{\mathbb{P}^{\mathfrak{s}}}^{\mathfrak{s p}{ }^{\dagger} \dagger}\left(\vec{p} ; \vec{\Lambda}^{*}\right)
$$

is an isomorphism.

First we start with the case $m=2$.

Proposition 6.2. If $m=2$, then the rank-level duality map (6.1) is an isomorphism.

Proof. When $m=2$, the dimension of the conformal block is one if $\Lambda^{(1) *}=\Lambda^{(2)}$, and zero otherwise. So we have only to prove that the rank-level duality map is non-zero if $\Lambda^{(1) *}=\Lambda^{(2)}$. By Proposition 4.2, it suffices to prove the corresponding strange duality map is non-zero.

For this, in view of the definition of the strange duality map, we have only to find a pair of parabolic symplectic bundles

$$
(\mathbb{E}, \mathbb{G}) \in M_{2 r}\left(\mathbb{P}^{1}, \vec{p} ; \mathcal{O}_{C}\right) \times M_{2 s}\left(\mathbb{P}^{1}, \vec{p} ; \omega_{\mathbb{P}^{1}}(\vec{p})\right)
$$


such that the canonical pfaffian section $\Theta$ does not vanish at the point $\tau_{\left(\mathbb{P}^{1} ; \vec{\Lambda}\right)}(\mathbb{E}, \mathbb{G}) \in$ $N_{4 r s}\left(\mathbb{P}^{1} ; \omega_{\mathbb{P}^{1}}\right)$.

Let

$$
E=\bigoplus_{i=1}^{2 r} \mathcal{O}_{\mathbb{P}^{1}} \cdot \mathbf{e}_{i} \quad \text { and } \quad G=\bigoplus_{i=1}^{2 s} \mathcal{O}_{\mathbb{P}^{1}} \cdot \mathbf{g}_{i}
$$

The symplectic forms are given by

$$
\begin{gathered}
\left(\mathbf{e}_{i}, \mathbf{e}_{j}\right)=\left(\mathbf{e}_{r+i}, \mathbf{e}_{r+j}\right)=0, \quad\left(\mathbf{e}_{i}, \mathbf{e}_{r+j}\right)=\delta_{i j} \quad 1 \leq i, j \leq r \\
\left(\mathbf{g}_{i}, \mathbf{g}_{j}\right)=\left(\mathbf{g}_{s+i}, \mathbf{g}_{s+j}\right)=0, \quad\left(\mathbf{g}_{i}, \mathbf{g}_{s+j}\right)=\delta_{i j} \quad 1 \leq i, j \leq s .
\end{gathered}
$$

We put

$$
\begin{aligned}
\mathbf{e}_{i}^{(1)}=\mathbf{e}_{i} \quad 1 \leq i \leq 2 r, \quad \mathbf{g}_{i}^{(1)}=\mathbf{g}_{i} \quad 1 \leq i \leq 2 s \\
\mathbf{e}_{i}^{(2)}=\mathbf{e}_{r+i}, \quad \mathbf{e}_{r+i}^{(2)}=\mathbf{e}_{i} \quad 1 \leq i \leq r \\
\mathbf{g}_{i}^{(2)}=\mathbf{g}_{s+i}, \quad \mathbf{g}_{s+i}^{(2)}=\mathbf{f}_{i} \quad 1 \leq i \leq s .
\end{aligned}
$$

For $j=1,2$, we define the the filtrations of $\left.E\right|_{p^{(j)}}$ and $\left.G\right|_{p^{(j)}}$ by

$$
\begin{aligned}
\left.E\right|_{p^{(j)}} & =\bigoplus_{i=1}^{2 r} \mathbb{C} \mathbf{e}_{i} \supset<\mathbf{e}_{1}^{(j)}, \ldots, \mathbf{e}_{r}^{(j)}>\supset \cdots \supset<\mathbf{e}_{1}^{(j)}, \mathbf{e}_{2}^{(j)}>\supset<\mathbf{e}_{1}^{(j)}>\supset 0 \\
\left.G\right|_{p^{(j)}}= & \bigoplus_{i=1}^{2 s} \mathbb{C} \mathbf{g}_{i} \supset<\mathbf{g}_{1}^{(j)}, \ldots, \mathbf{g}_{s}^{(j)}>\supset \cdots \supset<\mathbf{g}_{1}^{(j)}, \mathbf{g}_{2}^{(j)}>\supset<\mathbf{g}_{1}^{(j)}>\supset 0 .
\end{aligned}
$$

Let $\mathbb{E}$ and $\mathbb{G}$ be the parabolic symplectic bundles with these parabolic structures. Then the orthogonal bundle $\tau_{\left(\mathbb{P}^{1} ; \vec{\Lambda}\right)}(\mathbb{E}, \mathbb{G})$ becomes a direct sum of $\mathcal{O}_{\mathbb{P}^{1}}(-1)$ (cf. Figure 1 and 2), so it has no global sections.

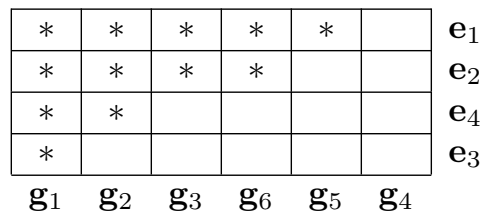

FigurE 1. The isotropic subspace $\mu_{\Lambda^{(1)}}\left(\left.E\right|_{p^{(1)} \bullet},\left.G\right|_{p^{(1)} \bullet}\right)$ for $(r, s)=(2,3)$ and $\Lambda^{(1)}=(2,1)$.

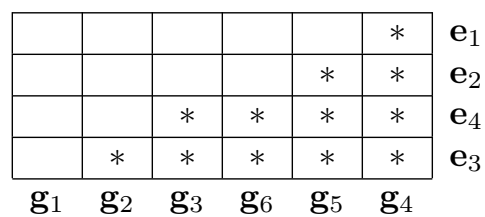

Figure 2. The isotropic subspace $\mu_{\Lambda^{(2)}}\left(\left.E\right|_{p^{(2)} \bullet},\left.G\right|_{p^{(2)} \bullet}\right)$ for $(r, s)=(2,3)$ and $\Lambda^{(1)}=(2,1)$.

This means that the canonical pfaffian section $\Theta$ does not vanish at $\tau_{\left(\mathbb{P}^{1} ; \vec{\Lambda}\right)}(\mathbb{E}, \mathbb{G})$.

Next we treat a special case with $m=4$. 
Proposition 6.3. Let $\left(\mathbb{P}^{1}, \vec{p}=\left(p^{(1)}, p^{(2)}, p^{(3)}, p^{(4)}\right)\right)$ be a 4-pointed $\mathbb{P}^{1}$, and $\vec{\Lambda}=$ $\left(\Lambda^{(1)}, \Lambda^{(2)}, \Lambda^{(3)}, \Lambda^{(4)}\right)$ a 4-tuple of Young diagrams of type $\leq(r, s)$ such that $\Lambda^{(3)}=$ $(1 \geq 0 \geq \cdots \geq 0)$ and $\Lambda^{(4)}=(0 \geq 0 \geq \cdots \geq 0)$. Assume that $\sum_{j=1}^{4}\left|\Lambda^{(j)}\right|$ is even.

Then the map (6.1) is an isomorphism.

Proof. By Proposition 4.2, it suffices to prove that the corresponding strange duality map is an isomorphism. By Remark 2.10, we may ignore the point $p^{(4)}$. In this case, by Proposition 3.5, the dimension of the target and the source of the duality map is one if $\Lambda^{(2)}$ is obtained from $\Lambda^{(1)}$ by adding or deleting one box, and zero otherwise. We may assume that $\Lambda^{(2)}$ is obtained from $\Lambda^{(1)}$ by adding one box in the $a$-th row from the top and in the $b$-th column from the left.

As in the proof of Proposition 6.2, we have only to find a pair of parabolic symplectic bundles $\left(\mathbb{E}, \mathbb{G}\right.$ ) on a 3 -pointed $\mathbb{P}^{1}$ such that the canonical pfaffian section $\Theta$ does not vanish at the point $\tau_{\left(\mathbb{P}^{1} ; \vec{\Lambda}\right)}(\mathbb{E}, \mathbb{G}) \in N_{4 r s}\left(\mathbb{P}^{1} ; \omega_{\mathbb{P}^{1}}\right)$.

Let

$$
E=\bigoplus_{i=1}^{2 r} \mathcal{O}_{\mathbb{P}^{1}} \cdot \mathbf{e}_{i} \quad \text { and } \quad G=\bigoplus_{i=1}^{s} \mathcal{O}_{\mathbb{P}^{1}} \cdot \mathbf{g}_{i} \oplus \bigoplus_{i=s+1}^{2 s} \mathcal{O}_{\mathbb{P}^{1}}(1) \cdot \mathbf{g}_{i}
$$

The symplectic forms are given by (6.2), and for $j=1,2, \mathbf{e}_{i}^{(j)}$ and $\mathbf{g}_{i}^{(j)}$ are defined by (6.3). We put

$$
\begin{aligned}
& \mathbf{e}_{1}^{(3)}=\mathbf{e}_{a}, \mathbf{e}_{a}^{(3)}=\mathbf{e}_{1}, \mathbf{e}_{r+1}^{(3)}=\mathbf{e}_{r+a}, \mathbf{e}_{r+a}^{(3)}=\mathbf{e}_{r+1}, \\
& \mathbf{e}_{i}^{(3)}=\mathbf{e}_{i} \text { for } \quad i \in\{1, \ldots, 2 r\} \backslash\{1, a, r+1, r+a\}, \\
& \mathbf{g}_{s+1-b}^{(3)}=\mathbf{g}_{s}, \mathbf{g}_{s}^{(3)}=\mathbf{g}_{s+1-b}, \mathbf{g}_{2 s+1-b}^{(3)}=\mathbf{g}_{2 s}, \mathbf{g}_{2 s}^{(3)}=\mathbf{g}_{2 s+1-b}, \\
& \mathbf{g}_{i}^{(3)}=\mathbf{g}_{i} \text { for } \quad i \in\{1, \ldots, 2 s\} \backslash\{s+1-b, s, 2 s+1-b, 2 s\} .
\end{aligned}
$$

We define the the filtrations of $\left.E\right|_{p^{(j)}}$ and $\left.G\right|_{p^{(j)}}$ by (6.4). If we let $\mathbb{E}$ and $\mathbb{G}$ be the parabolic symplectic bundles with these parabolic structures, then the orthogonal bundle $\tau_{\left(\mathbb{P}^{1} ; \vec{\Lambda}\right)}(\mathbb{E}, \mathbb{G})$ becomes again a direct sum of $\mathcal{O}_{\mathbb{P}^{1}}(-1)$.

In the next proposition we shall consider the $m$-pointed case where all the marked points except two of them are labeled by the Young diagrams $(0 \geq 0 \geq \cdots \geq 0)$ or $(1 \geq 0 \geq \cdots \geq 0)$.

Proposition 6.4. Let $\left(\mathbb{P}^{1}, \vec{p}\right)$ and $\vec{\Lambda}$ be as in Theorem 6.1. Assume that, for $3 \leq \forall j \leq m, \Lambda^{(j)}$ is either $(0 \geq 0 \geq \cdots \geq 0)$ (i.e. the empty Young diagram) or $(1 \geq 0 \geq \cdots \geq 0)$. Then the rank-level duality map (6.1) is an isomorphism.

Proof. By Corollary 4.4, we can add or delete points labeled by the empty Young diagram. If $\Lambda^{(j)}$ is the empty Young diagram for all $3 \leq j \leq m$, then the proposition follows from Proposition 6.2. We may assume that $m \geq 4$, and that for $3 \leq j \leq$ $m / 2+1, \Lambda^{(j)}$ is $(0 \geq 0 \geq \cdots \geq 0)$, and for $m / 2+2 \leq j \leq m,(1 \geq 0 \geq \cdots \geq 0)$. We proceed by induction on $m$.

If $m=4$, then the proposition follows from Proposition 6.3. Let $m \geq 6$. We make $\mathbb{P}^{1}$ degenerate to an $m$-pointed nodal curve $C_{1} \cup C_{2}$ with $C_{1}$ and $C_{2}$ isomorphic to $\mathbb{P}^{1}$ and intersecting transversely only one point $u$ such that under the 
degeneration, the points $p^{(1)}, p^{(3)}, p^{(m / 2+2)}$ on $\mathbb{P}^{1}$ specialize to points on $C_{1}$, denoted also by $p^{(1)}, p^{(3)}, p^{(m / 2+2)}$, and the other marked points to points on $C_{2}$.

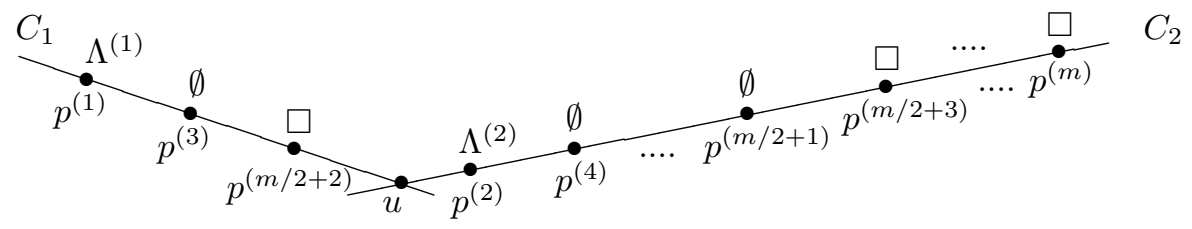

Put

$$
\begin{aligned}
& \overrightarrow{p_{1}}=\left(p^{(1)}, p^{(3)}, p^{(m / 2+2)}\right) \quad \text { and } \quad \overrightarrow{p_{2}}=\vec{p} \backslash \overrightarrow{p_{1}} \\
& \overrightarrow{\Lambda_{1}}=\left(\Lambda^{(1)}, \Lambda^{(3)}, \Lambda^{(m / 2+2)}\right) \quad \text { and } \quad \overrightarrow{\Lambda_{2}}=\vec{\Lambda} \backslash \overrightarrow{\Lambda_{1}} \text {. }
\end{aligned}
$$

By induction hypothesis, for any Young diagram $\Gamma$ of type $\leq(r, s)$ with $|\Gamma| \equiv$ $\sum_{j=1}^{3}\left|\Lambda^{(j)}\right|(\bmod 2)$, the rank-level duality maps

$$
\begin{gathered}
V_{C_{1} \mathfrak{s}^{\dagger}}^{\mathfrak{s p}_{2}}\left(\overrightarrow{p_{1}} \cup\{u\} ; \overrightarrow{\Lambda_{1}} \cup\{\Gamma\}\right)^{\vee} \rightarrow V_{C_{1}}^{\mathfrak{s p}_{2 s}^{\dagger}}\left(\overrightarrow{p_{1}} \cup\{u\} ;{\overrightarrow{\Lambda_{1}}}^{*} \cup\left\{\Gamma^{*}\right\}\right), \\
V_{C_{2}}^{\mathfrak{s p}_{2 r} \dagger}\left(\overrightarrow{p_{2}} \cup\{u\} ; \overrightarrow{\Lambda_{2}} \cup\{\Gamma\}\right)^{\vee} \rightarrow V_{C_{2}}^{\mathfrak{s p}_{2 s} \dagger}\left(\overrightarrow{p_{2}} \cup\{u\} ;{\overrightarrow{\Lambda_{2}}}^{*} \cup\left\{\Gamma^{*}\right\}\right)
\end{gathered}
$$

are isomorphisms.

By the compatibility of the rank-level duality and the factorization (Proposition 3.19), the rank-level duality map

$$
V_{C_{1} \cup C_{2}}^{\mathfrak{s p}_{2 r} \dagger}(\vec{p} ; \vec{\Lambda})^{\vee} \rightarrow V_{C_{1} \cup C_{2}}^{\mathfrak{s p}_{2 s} \dagger}\left(\vec{p} ; \vec{\Lambda}^{*}\right)
$$

is an isomorphism. Since the property that the rank-level duality map is an isomorphism is an open property, the proposition holds for a generic $m$-pointed $\mathbb{P}^{1}$. By Corollary 5.2, it holds for all $m$-pointed $\mathbb{P}^{1}$.

In the proof of Proposition 6.4, we derived the fact that the rank-level duality on the generic fiber is an isomorphism from the fact that it is an isomorphism on the special fiber. This was possible because the property that the morphism is an isomorphism is an open property.

Contrarily, in (one of the steps in) the proof of Theorem 6.1, we shall show that the rank-level duality map is an isomorphism on the special fiber by proving that it is an isomorphism on the generic fiber. Roughly speaking, we shall argue as follows. We consider a certain family $\mathcal{C} \rightarrow T$ of pointed nodal curves with labels by Young diagrams over a 2-dimensional scheme $T$ with a point $\mathbf{o} \in T$. On $T$, we have the family of rank-level duality maps

$$
V_{\mathcal{C} / T}^{\mathfrak{s p}_{2 r}^{\dagger}}(\vec{p} ; \vec{\Lambda})^{\vee} \rightarrow V_{\mathcal{C} / T}^{\mathfrak{s p}_{2 s}^{\dagger}}\left(\vec{p} ; \vec{\Lambda}^{*}\right)
$$

between the families of conformal blocks. We prove that it is an isomorphism over $T \backslash\{\mathbf{o}\}$. From this it follows that the rank-level duality map must be an isomorphism over the point $\mathbf{o}$ as well, taking into account the fact that the family of conformal blocks is a vector bundle on $T$ by virtue of [TUY].

In the proof of Theorem 6.1 we need a family $\mathcal{C} \rightarrow T$ of nodal curves of arithmetic genus zero with sections $q^{(1)}, \ldots, q^{(a)}, r^{(1)}, \ldots, r^{(b)}, s^{(1)}, s^{(2)}$ having the following properties $(\boldsymbol{\Lambda})$ :

- For each $t \in T$, the points $q_{t}^{(1)}, \ldots, q_{t}^{(a)}, r_{t}^{(1)}, \ldots, r_{t}^{(b)}, s_{t}^{(1)}, s_{t}^{(2)} \in \mathcal{C}_{t}$ are distinct smooth points.

- $T$ is a variety of dimension 2 . There are curves $D_{1}$ and $D_{2}$ on $T$ with the following properties.

- For each $t \in T \backslash\left(D_{1} \cup D_{2}\right), \mathcal{C}_{t}$ is smooth, i.e., isomorphic to $\mathbb{P}^{1}$. 
- For each $t \in D_{1} \backslash D_{2}, \mathcal{C}_{t}=C_{1} \cup C_{2}$ with $C_{i}$ isomorphic to $\mathbb{P}^{1}, q^{(i)}, s^{(i)} \in C_{1}$ and $r^{(i)} \in C_{2}$.

- For each $t \in D_{2} \backslash D_{1}, \mathcal{C}_{t}=C_{1} \cup C_{2}$ with $C_{i}$ isomorphic to $\mathbb{P}^{1}, q^{(i)} \in C_{1}$ and $s^{(i)}, r^{(i)} \in C_{2}$.

- For each $t \in D_{1} \cap D_{2}, \mathcal{C}_{t}=C_{1} \cup C_{2} \cup C_{3}$ with $C_{i}$ isomorphic to $\mathbb{P}^{1}, C_{i} \cap C_{j} \neq \emptyset$ if and only if $|i-j| \leq 1, q^{(i)} \in C_{1}, s^{(i)} \in C_{2}$ and $r^{(i)} \in C_{3}$.

Such a family can be constructed explicitly by blowing up $\mathbb{P}^{1} \times \mathbb{A}^{2}$ suitably (cf. Remark 6.5).

Now let us move on to the proof of Theorem 6.1.

Proof of Theorem 6.1. The first step is the most essential part of the proof.

Step 1. We prove the theorem when $m=4$ and $\Lambda^{(4)}$ is the empty Young diagram.

Fix odd integers $a$ and $b$ with $a \geq\left|\Lambda^{(1)}\right|$ and $b \geq\left|\Lambda^{(2)}\right|$. Let

$$
\left(\mathcal{C} \stackrel{\pi}{\rightarrow} T, q^{(j)}(1 \leq j \leq a), r^{(j)}(1 \leq j \leq b), s^{(1)}, s^{(2)}\right)
$$

be a family of nodal curves of arithmetic genus zero and its sections having the properties ( $)$ above. We label the sections $q^{(j)}, r^{(j)}, s^{(j)}$ by Young diagrams $\Lambda_{q}^{(i)}$, $\Lambda_{r}^{(i)}, \Lambda_{s}^{(i)}$ of type $\leq(r, s)$ as follows:

$$
\begin{gathered}
\Lambda_{q}^{(i)}= \begin{cases}(1 \geq 0 \geq \cdots \geq 0) & \text { for } 1 \leq i \leq\left|\Lambda^{(1)}\right| \\
(0 \geq 0 \geq \cdots \geq 0) & \text { for }\left|\Lambda^{(1)}\right|<i \leq a,\end{cases} \\
\Lambda_{r}^{(i)}= \begin{cases}(1 \geq 0 \geq \cdots \geq 0) & \text { for } 1 \leq i \leq\left|\Lambda^{(2)}\right| \\
(0 \geq 0 \geq \cdots \geq 0) & \text { for }\left|\Lambda^{(2)}\right|<i \leq b,\end{cases} \\
\Lambda_{s}^{(1)}=\Lambda^{(3)} \text { and } \Lambda_{s}^{(2)}=\Lambda^{(4)}(=(0 \geq \cdots \geq 0)) .
\end{gathered}
$$

On $T$, we have a family of rank-level duality maps

$$
\rho: V_{\mathcal{C} / T}^{\mathfrak{s p}_{2}{ }^{\dagger}}\left(\vec{q} \cup \vec{r} \cup \vec{s} ; \overrightarrow{\Lambda_{q}} \cup \overrightarrow{\Lambda_{r}} \cup \overrightarrow{\Lambda_{s}}\right)^{\vee} \rightarrow V_{\mathcal{C} / T}^{\mathfrak{s p}_{2 s} \dagger}\left(\vec{q} \cup \vec{r} \cup \vec{s} ; \overrightarrow{\Lambda_{q}}{ }^{*} \cup \overrightarrow{\Lambda_{r}}{ }^{*} \cup \overrightarrow{\Lambda_{s}^{*}}\right) .
$$

For each $t \in T \backslash\left(D_{1} \cup D_{2}\right), \mathcal{C}_{t}$ is smooth, hence $\rho_{t}$ is an isomorphism by Proposition 6.4. For $t \in D_{1} \backslash D_{2}$, we have $\mathcal{C}_{t}=C_{1} \cup C_{2}$ with $q^{(i)}, s^{(i)} \in C_{1}$ and $r^{(i)} \in C_{2}$. Put $\{u\}:=C_{1} \cap C_{2}$, and let $\Gamma$ be a Young diagram of type $\leq(r, s)$ with $|\Gamma| \equiv\left|\Lambda^{(2)}\right|$ $(\bmod 2)$. Then for both pointed nodal curves with labeling by Young diagrams

$$
\left(C_{1} ; \vec{q} \cup \vec{s} \cup\{u\} ; \overrightarrow{\Lambda_{q}} \cup \overrightarrow{\Lambda_{s}} \cup\{\Gamma\}\right) \text { and }\left(C_{2} ; \vec{r} \cup\{u\} ; \overrightarrow{\Lambda_{r}} \cup\{\Gamma\}\right),
$$

the rank-level duality maps are isomorphisms by Proposition 6.4. From this and the compatibility of the rank-level duality map and the factorization, it follows that $\rho_{t}$ is an isomorphism. Likewise for $t \in D_{2} \backslash D_{1}, \rho_{t}$ is an isomorphism. Then $\rho$ must be an isomorphism because $\rho$ is an isomorphism outside of the codimension 2 subset $D_{1} \cap D_{2}$ and the families of conformal blocks are vector bundles by virtue of [TUY].

For $t \in D_{1} \cap D_{2}$, we have $\mathcal{C}_{t}=C_{1} \cup C_{2} \cup C_{3}$ with $q^{(i)} \in C_{1}, s^{(i)} \in C_{2}$ and $r^{(i)} \in C_{3}$. Put $\{u\}:=C_{1} \cap C_{2}$ and $\left\{u^{\prime}\right\}:=C_{2} \cap C_{3}$. The rank-level duality map $\rho_{t}$ is, by the factorization, a direct sum of tensor products of the rank-level duality maps

$$
\begin{aligned}
& V_{C_{1}}^{\mathfrak{s p}_{2 r} \dagger}\left(\vec{q} \cup\{u\} ; \overrightarrow{\Lambda_{q}} \cup\{\Gamma\}\right)^{\vee} \rightarrow V_{C_{1}}^{\mathfrak{s p}_{2} \dagger}\left(\vec{q} \cup\{u\} ;{\overrightarrow{\Lambda_{q}}}^{*} \cup\left\{\Gamma^{*}\right\}\right), \\
& V_{C_{2}}^{\mathfrak{s p}_{2 r} \dagger}\left(\vec{s} \cup\left\{u, u^{\prime}\right\} ; \overrightarrow{\Lambda_{s}} \cup\left\{\Gamma, \Gamma^{\prime}\right\}\right)^{\vee} \rightarrow V_{C_{2}}^{\mathfrak{s p}_{2 s} \dagger}\left(\vec{s} \cup\left\{u, u^{\prime}\right\} ;{\overrightarrow{\Lambda_{s}}}^{*} \cup\left\{\Gamma^{*}, \Gamma^{\prime *}\right\}\right), \\
& V_{C_{3}}^{\mathfrak{s p} p_{2 r}^{\dagger}}\left(\vec{r} \cup\left\{u^{\prime}\right\} ; \overrightarrow{\Lambda_{r}} \cup\left\{\Gamma^{\prime}\right\}\right)^{\vee} \rightarrow V_{C_{3}}^{\mathfrak{s p} p_{2 s}^{\dagger}}\left(\vec{r} \cup\left\{u^{\prime}\right\} ;{\overrightarrow{\Lambda_{r}}}^{*} \cup\left\{\Gamma^{\prime *}\right\}\right),
\end{aligned}
$$

where $\Gamma$ and $\Gamma^{\prime}$ are Young diagrams of type $\leq(r, s)$ such that $|\Gamma| \equiv\left|\Lambda^{(1)}\right|(\bmod 2)$ and $\left|\Gamma^{\prime}\right| \equiv\left|\Lambda^{(2)}\right|(\bmod 2)$. Since $\rho_{t}$ is an isomorphism, each direct summand, the tensor product of the above three rank-level duality maps, is an isomorphism. In 
particular, take $\Gamma=\Lambda^{(1)}$ and $\Gamma^{\prime}=\Lambda^{(2)}$. Then conformal blocks appearing in (6.5) and (6.7) are nonzero. (This follows from Proposition 3.5 using the fusion rule of dimensions of conformal blocks.) Hence the map (6.6) must be an isomorphism.

Step 2. We prove the theorem when $m=4$.

Take extra points $p^{(5)}, p^{(6)} \in \mathbb{P}^{1} \backslash \vec{p}$ and label them by the empty Young diagram. We make $\mathbb{P}^{1}$ degenerate to a nodal curve $C_{1} \cup C_{2}$ with $C_{i}$ isomorphic to $\mathbb{P}^{1}$ such that the points $p^{(1)}, p^{(2)}, p^{(5)}$ on $\mathbb{P}^{1}$ specialize to points on $C_{1}$, and $p^{(3)}, p^{(4)}, p^{(6)}$ on $\mathbb{P}^{1}$ to points on $C_{2}$. By abuse of notation, the specialization of the point $p^{(i)} \in \mathbb{P}^{1}$ to $C_{1} \cup C_{2}$ is also denote by $p^{(i)}$. Put $\{u\}:=C_{1} \cap C_{2}$.

By Step 1, for a Young diagram $\Gamma$ of type $\leq(r, s)$ with $|\Gamma| \equiv \sum_{i=1,2}\left|\Lambda^{(i)}\right|(\bmod 2)$, the rank-level duality maps for the 4 -pointed $\mathbb{P}^{1}$ with labeling by Young diagrams

$$
\begin{aligned}
& \left(C_{1} ;\left(p^{(1)}, p^{(2)}, p^{(5)}, u\right) ;\left(\Lambda^{(1)}, \Lambda^{(2)}, \emptyset, \Gamma\right)\right), \\
& \left(C_{2} ;\left(p^{(3)}, p^{(4)}, p^{(6)}, u\right) ;\left(\Lambda^{(3)}, \Lambda^{(4)}, \emptyset, \Gamma\right)\right)
\end{aligned}
$$

are isomorphisms. hence again by Proposition 3.19, the rank-level duality map for

$$
\left(C_{1} \cup C_{2} ; \vec{p} \cup\left\{p^{(5)}, p^{(6)}\right\} ; \vec{\Lambda} \cup\{\emptyset, \emptyset\}\right)
$$

is an isomorphism. So the rank-level duality map for

$$
\left(\mathbb{P}^{1} ; \vec{p} \cup\left\{p^{(5)}, p^{(6)}\right\} ; \vec{\Lambda} \cup\{\emptyset, \emptyset\}\right)
$$

is an isomorphism if ( $\left.\mathbb{P}^{1} ; \vec{p} \cup\left\{p^{(5)}, p^{(6)}\right\}\right)$ is generic. By Corollary 4.4, the rank-level duality map for a generic 4-pointed $\mathbb{P}^{1}$ is an isomorphism. By Corollary 5.2, it is an isomorphism for all 4-pointed $\mathbb{P}^{1}$.

Step 3. The general case follows again by degeneration method and induction on $m$. Make $\left(\mathbb{P}^{1} ; \vec{p}\right)$ degenerate to a pointed nodal curve $\left(C_{1} \cup C_{2} ; \vec{p}\right)$ with $C_{i}$ isomorphic to $\mathbb{P}^{1}$ such that $p^{(1)}, p^{(2)}, p^{(3)} \in C_{1}$ and $p^{(j)} \in C_{2}$ for $4 \leq j \leq m$. Then argue as in Step 2.

Remark 6.5. We remark that a family $\mathcal{C} \rightarrow T$ of nodal curves of arithmetic genus zero together with sections $q^{(1)}, \ldots, q^{(a)}, r^{(1)}, \ldots, r^{(b)}, s^{(1)}, s^{(2)}$ having the properties ( $)$ can be constructed explicitly by blowing up $\mathbb{P}^{1} \times \mathbb{A}^{2}$ suitably.

Let $p r: \mathbb{P}^{1} \times \mathbb{A}^{2} \rightarrow \mathbb{A}^{2}$ be the second projection. Let $\left(z_{1}, z_{2}\right)$ be the affine coordinate of $\mathbb{A}^{2}, u$ be the affine coordinate of $\mathbb{A}^{1} \subset \mathbb{P}^{1}=\mathbb{A}^{1} \cup\{\infty\}$. Put $u^{\prime}=u-1$. Let $\left\{\alpha_{1}, \ldots, \alpha_{a}\right\},\left\{\beta_{1}, \ldots, \beta_{b}\right\}$ and $\left\{\gamma_{1}, \gamma_{2}\right\}$ be sets of distinct complex numbers with $\gamma_{i} \neq 0,1$ for $i=1,2$. We define closed subschemes of $\mathbb{P}^{1} \times \mathbb{A}^{2}$ by

$$
\begin{aligned}
L_{1}: u=z_{1}=0, & L_{2}: u^{\prime}=z_{2}=0, & \\
\bar{q}^{(i)}: u^{\prime}=\alpha_{i} z_{2}, & \bar{r}^{(i)}: u=\beta_{i} z_{1}, & \bar{s}^{(i)}: u=\gamma_{i} .
\end{aligned}
$$

Let $b l: \mathcal{B} \rightarrow \mathbb{P}^{1} \times \mathbb{A}^{2}$ be the blowing-up along the subscheme $L_{1} \sqcup L_{2}$. Let $q^{(i)}, r^{(i)}, s^{(i)}$ be the strict transforms in $\mathcal{B}$ of $\bar{q}^{(i)}, \bar{r}^{(i)}, \bar{s}^{(i)}$. We denote by $\pi$ the composite $p r \circ b l: \mathcal{B} \rightarrow \mathbb{A}^{2}$. Then over the origin $\mathbf{o} \in T, q^{(i)}, r^{(i)}, s^{(i)}$ give distinct smooth points of the fiber. Take a Zariski open neighborhood $\mathbf{o} \in T \subset \mathbb{A}^{2}$ small enough that for each $t \in T, q^{(i)}, r^{(i)}, s^{(i)}$ give distinct smooth points of the fiber $\mathcal{B}_{t}$. If we let $D_{i}:=\left\{z_{i}=0\right\} \subset T$, then the family $\mathcal{C}:=\pi^{-1}(T) \rightarrow T$ has the properties

\section{REFERENCES}

[A] T. Abe: Degeneration of the strange duality map for symplectic bundles, to appear in Journal für die reine und angewandte Mathematik.

[B96] A. Beauville: Conformal blocks, fusion rules and the Verlinde formula, Proceedings of the Hirzebruch 65 Conference on Algebraic Geometry (Ramat Gan, 1993), 75-96, Israel Math. Conf. Proc., 9, Bar-Ilan Univ., Ramat Gan, 1996. 
[B06] A. Beauville: Orthogonal bundles on curves and theta functions, Ann. Inst. Fourier (Grenoble) 56 (2006), no. 5, 1405-1418.

[Bel07] P. Belkale: Strange duality and the Hitchin/WZW connection, arXiv:0705.0717.

[Bel08] P. Belkale: The strange duality conjecture for generic curves, J. Amer. Math. Soc. 21 (2008), no. 1, 235-258 (electronic).

[FH] W. Fulton and J.Harris: Representation theory. A first course. Graduate Texts in Mathematics, 129. Readings in Mathematics. Springer-Verlag, New York, 1991. xvi+551 pp.

[Has] K.Hasegawa: Spin module versions of Weyl's reciprocity theorem for classical Kac-Moody Lie algebras-an application to branching rule duality, Publ. Res. Inst. Math. Sci. 25 (1989), no. 5, 741-828.

[KZ] V. G. Knizhnik and A. B. Zamolodchikov: Current algebra and Wess-Zumino model in two dimensions, Nuclear Phys. B 247 (1984), no. 1, 83-103.

[L-S] Y. Laszlo and C. Sorger: The line bundles on the moduli of parabolic G-bundles over curves and their sections, Ann. Sci. Ecole Norm. Sup. (4) 30 (1997), no. 4, 499-525.

[L] P. Littelmann: A generalization of the Littlewood-Richardson rule, J. Algebra 130 (1990), no. $2,328-368$.

[M-O] A. Marian and D. Oprea: The level-rank duality for non-abelian theta functions, Invent. Math. 168 (2007), no. 2, 225-247.

[NT] T. Nakanishi and A. Tsuchiya: Level-rank duality of WZW models in conformal field theory, Comm. Math. Phys. 144 (1992), no. 2, 351-372.

[S] C. Sorger: La formule de Verlinde, Seminaire Bourbaki, Vol. 1994/95. Asterisque No. 237 (1996), Exp. No. 794, 3, 87-114.

[TUY] A. Tsuchiya, K. Ueno and Y. Yamada: Conformal field theory on universal family of stable curves with gauge symmetries, Integrable systems in quantum field theory and statistical mechanics, 459-566, Adv. Stud. Pure Math., 19, Academic Press, Boston, MA, 1989.

Research Institute for Mathematical Sciences, Kyoto University, Kitashirakawa, SAKYO-KU, KYOTO, 606-8502, JAPAN

E-mail address: abeken@kurims.kyoto-u.ac.jp 